\title{
A General Methodology for Structural Shape Optimization Problems Using Automatic Adaptive Remeshing
}

G. Bugeda x. Oliver 


\section{A General Methodology for Structural Shape Optimization Problems Using Automatic Adaptive Remeshing}
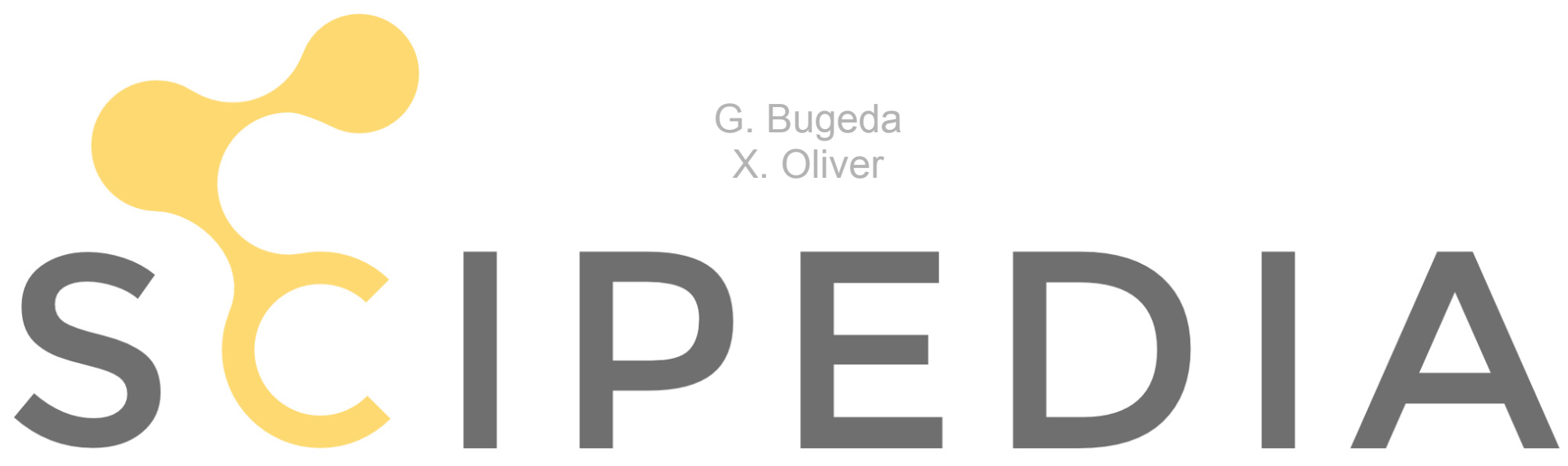

Register for free at https//www.scipedia.com to download the version without the watermark

Publication CIMNE Nº-19, Marzo 1992 


\section{SUMMARY}

The proposed methodology lies on the use of the adaptive mesh remeshing (AMR) techniques in the context of $2 \mathrm{D}$ shape optimization problems analyzed by the Finite Element Method. A suitable and very general technique for the parametrization of the optimization problem, that uses B-splines to define the boundary, is first presented. Then, mesh generation, using the advancing frontal method, the error estimator and the remeshing criteria are studied in the context of shape optimization problems. Particularly, the analytical sensitivity analysis of the different items ruling the problem (B-splines, Finite Element Mesh, structural behaviour, and error estimator) is studied in detail. The sensitivities of the Finite Flement Mesh and error estimetor permit their projection from one design to the next one leading to an "a priori knowledge" of the finite element error distribution on the new design without the necessity of any additional structural analysis. With this information the remeshing criteria permits to build up a finite element mesh on the new design with an specified and controlled level of error. The robustness and feliability of the proposed methodology is checked through several examples.
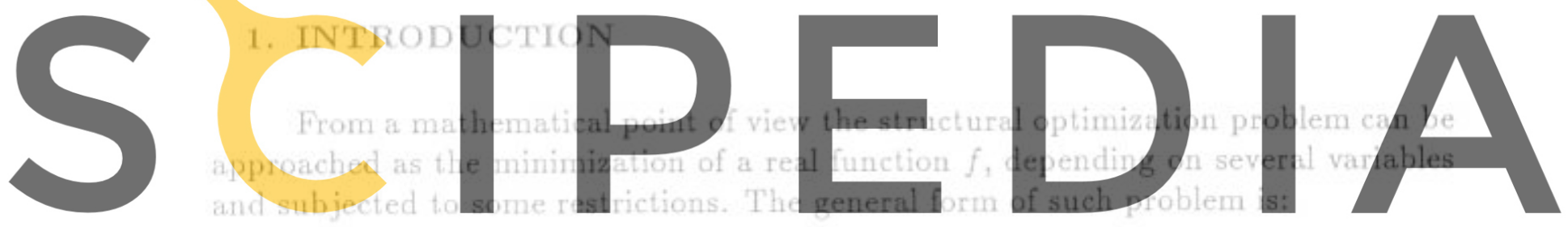

Register for free at https//www.scipedia.com to download the version without the watermark

$$
\text { with: } \begin{aligned}
& g(x)=\left\{g_{j}(x)\right\} ; j=1, \ldots, m \\
\text { verifying: } & g_{j}(\boldsymbol{x}) \leq 0 \quad ; j=1, \ldots, m \\
& a_{i} \leq x_{i} \leq b_{i} \quad ; i=1, \ldots, n
\end{aligned}
$$

where $f$ is the objective function, $x_{i}$ are the design variables and $g_{j}$ are inequality restrictions normally expressed in terms of stresses or displacements. The values $a_{i}$ and $b_{i}$ define lateral restrictions. Each set of values $x$ defines one structural design and the problem consists in finding those $x$ values defining the optimum design.

The algorithms for the resolution of eq. (1) are, normally, iterative and the computation of the derivatives (sensitivities) of the objective function and restrictions with respect to the design variables is needed. Besides, in each step of that process it is necessary to calculate the $f$ and $g$ values and its sensitivities. In many cases, as the ones considered in this work, those computations are done via a finite element analysis which allows us to know the structural response of each design, and compute the corresponding sensitivities. The definition of each design in terms of the $\boldsymbol{x}$ variables of eq. (1) is called the "parametrization" of the optimum design problem. 
There are many existing codes and different methodologies to solve the optimization problem of eq. (1) [5]. However, some problems still remain unsolved in this context ie: the inclusion of robust parametrization procedures for the definition of each design, the control of the error associated to the finite element computations and its influence on the solution of the optimization problem. Normally, once the optimization process is finished there is no warranty about the accuracy of the final design; sometimes a more accurate analysis would reveal that the final design is infeasible, violating one or more of the imposed restrictions.

Looking at this problem a general methodology to solve structural shape optimization problems should include the following features:

- General parametrigation nrocedures in order to be able to deal with different structural types with the same structural optimization code. The definition of any design should only need a reduced number of design variables.

An easy treatment of boundary conditions and loads.

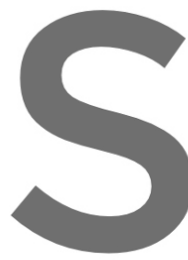

An easy and general definition of the objective function and restrictions.

An automatic, robust and flexible mesh generator.
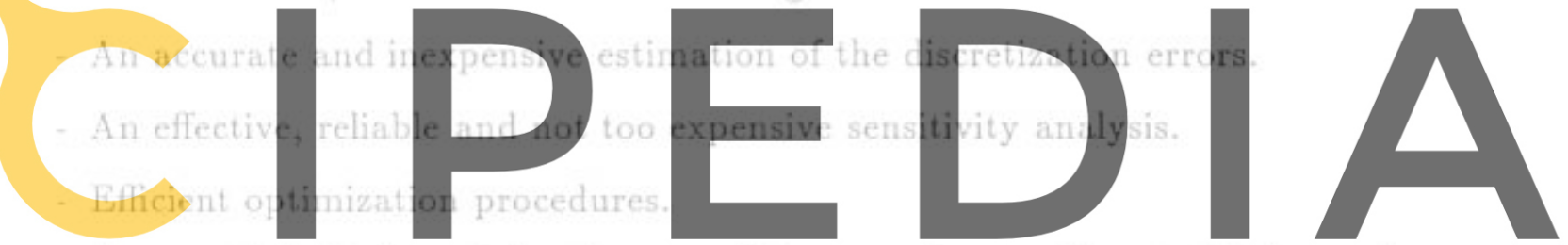

Automatic inclusion of adaptive remeshing procedures without a big increasing

Register for free at https//www.scipedia.com to download the version without the watermark

A control over the quality of the meshes used for each design, ie: distorted elements should be avoided when big changes of the structural shape are expected.

In this paper we present a general methodology for structural optimization problems including all the mentioned features. In the following sections we describe the parametrization of the optimum design problem, automatic mesh generation, error estimation, sensitivity analysis, and the adaptive remeshing strategy which is used in this methodology.

\section{THE PROPOSED METHODOLOGY}

A general scheme for the proposed methodology is shown in Figure 1. It consists in a series of modulus each one corresponding to a specific task.

The input module reads the data concerning parametrization of the problem, the objective function, design variables and restrictions defining the structural optimization problem (1) to be solved. 
After the input module there is a closed loop for each iteration of the optimization process. Each iteration corresponds to one design that is called the actual design.

In the mesh parameters module an AMR strategy defines the characteristics of a proper mesh to be used in the computations on the actual design. The proposed AMR strategy is based on the results of the previous design. For the first design a uniform mesh has to be specified because no previous information is available.

The mesh generation module uses the information coming from the previous module to generate a mesh for the actual design. Traditionally, structured meshes are used for the optimization processes and then a good control on the mesh quality is not possible. In the present methodology we propose to use a non structured mesh generator. The module should carry out not only the mesh generation but also the application of the specified boundary conditions and loads. Moreover, it has also to produce the necessary information to compute the mesh sensitivity analysis in a posterior module.

The structural analysis module performs the classical structural Finite Element (FE) computations. The generated information about displacements and
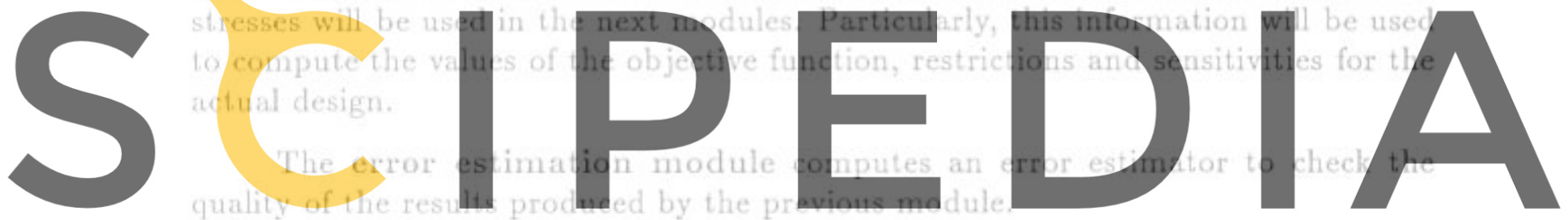

Register for free at https//www.scipedia.com to download the wersion without the watermark of the shape optmization problem. Some of the main newness of the proposed methodology are included in this module. The objective function and restrictions are normally expressed in terms of the volume, shape, and structural behaviour. Thus, their sensitivity analysis needs the derivation of each intermediate variable involved in the FE analysis. In particular, sensitivities of the following variables are computed: boundary shape (parametrization), nodal coordinates (mesh sensitivity analysis), displacements, stresses at gauss points, nodal stresses, objective function, restrictions and error estimator.

In the present work first and second order sensitivities are computed. Although the sensitivity analysis of the error estimator is not strictly necessary to solve the optimization problem, it is very useful for the AMR strategy this being one of the main characteristics of the proposed methodology. Another important feature of this module is the sensitivity analysis of the nodal coordinates of a non structured mesh as will be described later.

The design improvement module uses the previous information to produce an improved design. Using the sensitivity analysis, the error estimator and other magnitudes of the actual design are projected to the next one in order to produce the 
necessary information for the mesh parameters module. This projection is the main newness of this methodology because it allows to introduce an AMR strategy without remeshing any design. It saves a lot of CPU time because the adaptive remeshing is introduced without repeating FE computations over any design. In that sense, an "a posteriori" error estimator for the previous design is projected and converted in an "a priori" error estimator for the new one.

After checking the convergence (minimum condition) of the optimization process, a new loop starts for the next design.

In the next sections some of those modules are commented in more detail.

\section{PARAMETRTZATION OF THE STRUCTURAL OPTMMUM DESIGN PROBLEM}
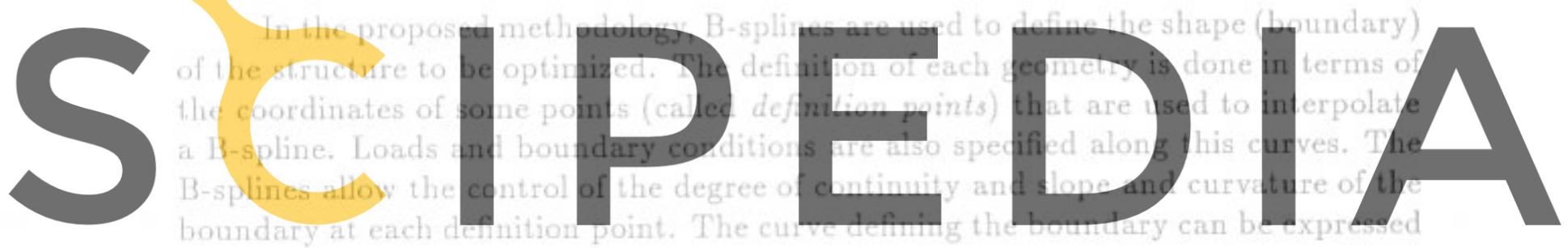

Register for free at https//www.scipedia.com to download the version without the watermark

$$
\boldsymbol{r}(t)=\sum_{l=0}^{q} \boldsymbol{r}_{l} N_{4, t+1}(t)
$$

where $\boldsymbol{r}(t)$ is the position vector depending on the $t$ parameter. The curve is expressed as a lineal combination of $q+1$ normalized fourth order (cubic) B-splines [3]. The $r_{l}$ coefficients are the coordinates of the so called polygon definition points ${ }^{[3]}$ and are found using the coordinates of the definition points and some additional conditions on them. The normal degree of continuity of a cubic B-spline is $C^{2}$, but it can be decreased at any definition point through one additional condition for each diminished degree. This additional conditions are normally related with slopes $p$ and curvatures of the curve over the corresponding point. The definition of the slope of the curve at a definition point requires also the specification of an additional factor $f$ for this point. From eq. (2) the coordinates of the definition points and the additional conditions are used to form a lineal system of equations:

$$
V=N R
$$


where $V$ is a vector composed by the imposed conditions at the definition points, the $N$ matrix contains some terms corresponding to the values of the polynomials $N$ that define each $\mathrm{B}$-spline, and the $R$ vector contains the coefficients $\boldsymbol{r}_{i}$ to be computed. Details of this process can be found in [3].

The definition of the geometry of each design in terms of the coordinates of some definition points, and the characteristics of the interpolating curve is very useful in the structural optimization problems because after defining an initial design in terms of some definition points, some of their characteristics (coordinates, slopes $p$, factors $f$, etc.) can be used as design variables. The values corresponding to a boundary condition, material properties, and load multiplication factors can be used as design variables as well. Thus, this definition can be used to parametrize the structural optimization problem in a very general form.

Restrictions $g_{j}$ of eq. (1) can also be defined along the curves defining the boundary. Reslrictions on displacements and stresses can be imposed at points located on that curves or along complete parts of them. It is worth noting that the maximum values of displacements and stresses are normally produced along the boundary, this emphaticing the importance of being able to impose restrictions on
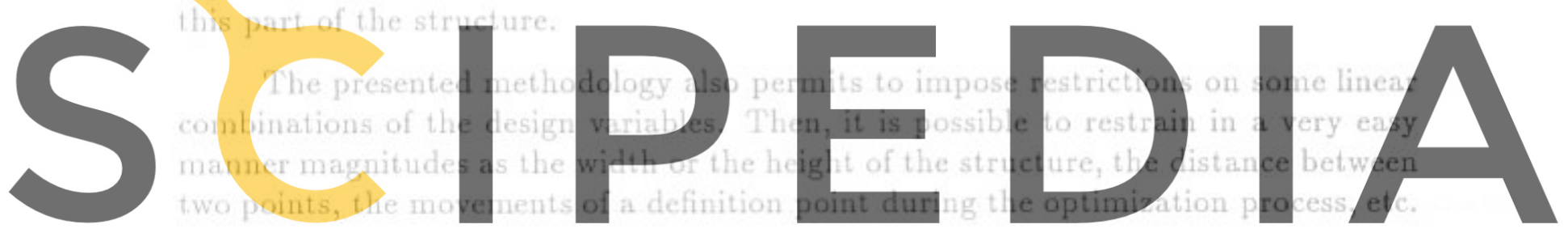

Register for free at https//www.scipedia.com to download the version without the watermark

After the geometric definition of each design it is necessary to generate a finite element mesh. To do that, an advancing front technique, which allows the control of the element size at every point of an arbitrarily shaped domain, is used. This method produces non structured triangular meshes. Details of this generation algorithm can be found in [3] and [7-8].

The information about the characteristics of the desired mesh is stored in the called background grid. That is a triangular mesh which completely covers the domain of interest. This background mesh is used to provide a spatial distribution for the sizes $\delta$ of the elements of the desired mesh. During the generation process the local value of the element size is obtained from the information in the background grid.

For the first design there is no information to predict the sizing of the mesh, so an initial background grid has to be constructed by hand and a uniform sizing can be specified. For next designs the background grid can be constructed with the information coming from the previous ones.

At the beginning of the generation process the boundary of the domain is 
discretized (see Figure 2.). Nodes are placed on the boundary with a separation between them corresponding to the spacing $\delta$ specified in the background grid. Each pair of contiguous nodes are joined by a straight line in order to form the initial generation front.

For the generation of every new triangle a segment is chosen from the generation front and the new triangle is built containing the segment as one of its sides. The new element is formed generating a new node or connecting the segment of the front with an already existing node. After the generation of each new element it is necessary to update the generation front in order to get all the segments over which new elements can be built. The process stops when there are no segments in the front.

The steps to be followed to generate a new element with size $\delta$ are (see Figure 3)
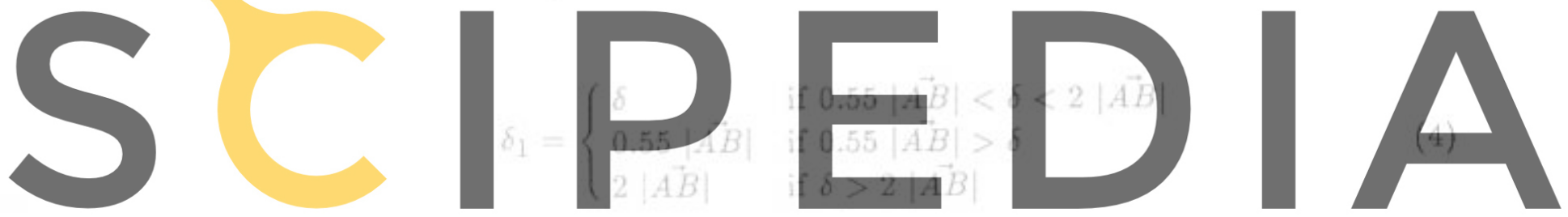

Register for free at https//www.scipedia.com to download the version without the watermark 3. Position the points $C_{2}, C_{3}, C_{4}, C_{5}, C_{6}$ in a equidistant form in the segment $C_{1} M$.

4. Make a list of the points of the actual generation front which lie inside a circie of radius $r=3|\overrightarrow{A B}|$ and center in $C_{1}$. This list is ordered according to the distance to the point $C_{1}$ beginning with the closest one.

5. Position the points $C_{1}, \ldots, C_{6}$ in the list adding a penalty of $\delta_{1}$ to their distance to $C_{1}$.

6. Search the connection point $C$. It will be the first node of the list which accomplish the next two conditions:

A. The interior of the triangle $A B C$ does not contain any other nodes of the list (excepting $C_{1}, \ldots, C_{6}$ ).

B. The segment $C M$ does not cut any of the segments in the actual generation front.

7. Update the generation front (see Figure 2). If it is void the process is finished.

With this algorithm the coordinates of each point $C$ are obtained from the coordinates of the two previous points $A$ and $B$ using the expression: 


$$
r_{C}=\left(\frac{x_{A}+x_{B}}{2}, \frac{y_{A}+y_{B}}{2}\right)+\frac{(7-i) \delta_{1} \sqrt{3}}{12} \frac{\left(y_{B}-y_{A}, x_{A}-x_{B}\right)}{\sqrt{\left(y_{B}-y_{A}\right)^{2}+\left(x_{B}-x_{A}\right)^{2}}}
$$

where $\delta$ is the value for the required element size, interpolated from the background grid which in turn is used to define the characteristics of the new mesh, and $i$ range from 1 to 6 depending of the type of point $\left(C_{1}, \ldots, C_{6}\right)$.

\section{STRUCTURAL ANALYSIS}

The structural analysis of each design is computed using a bidimensional linear elastic model. It is a standard F.E.M. analysis to solve a linear elliptic problem of the form $\mid 10\}$;

$$
L_{u} \equiv S^{T} D S u=f \text { in } \Omega
$$
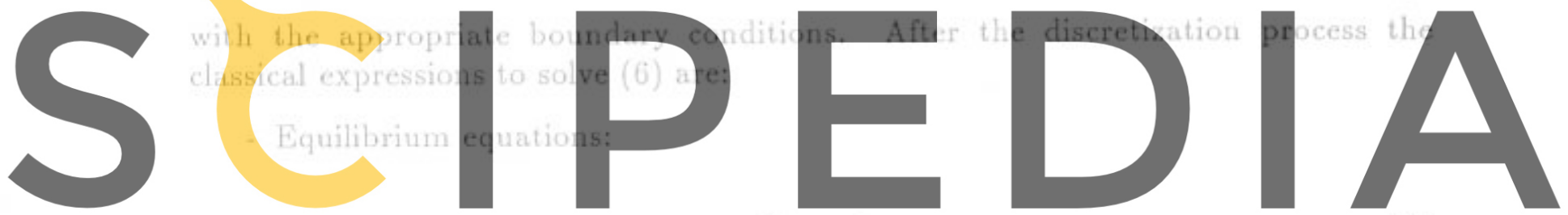

$K a=f$

Register for free at https//www.scipedia.com to download the version without the watermark

Assembling rules:

$$
\left\{\begin{array}{l}
K=\sum_{e} K_{e} \\
f=\sum_{e} f_{e} \\
f_{e}=f_{\Omega_{e}}+f_{\Sigma_{e}}+f_{P_{e}}
\end{array}\right.
$$

- Elemental stiffness matrix:

$$
K_{e}=\int_{\Omega_{e}} B^{T} D B d \Omega
$$

- Nodal forces vectors:

$$
\left\{\begin{array}{l}
f_{\Omega}=\int_{\Omega_{e}} N^{T} b d \Omega \\
f_{\Gamma_{e}}=\int_{\Gamma_{e}} N^{T} t d \Gamma \\
f_{P_{e}}=\sum N^{T} p
\end{array}\right.
$$


- Geometric and constitutive equations:

$$
\left\{\begin{array}{l}
\varepsilon=B a \\
\sigma=D \varepsilon
\end{array}\right.
$$

- Smoothing of stresses:

$$
\left\{\begin{array}{l}
\sigma^{*}=\sum N_{i} \bar{\sigma}_{i}^{*}=\bar{N}^{T} \overline{\boldsymbol{\sigma}}^{*} \\
\overline{\boldsymbol{\sigma}}^{*}=M^{-1} \Phi \\
\Phi=\sum_{e} \int_{\Omega_{e}} \bar{N}^{T} \boldsymbol{\sigma d} \Omega \\
\mathbb{M}=\sum_{e} M_{e} \\
M_{e}=\int_{\Omega_{e}} \bar{N} \bar{N}^{T} d \Omega
\end{array}\right.
$$
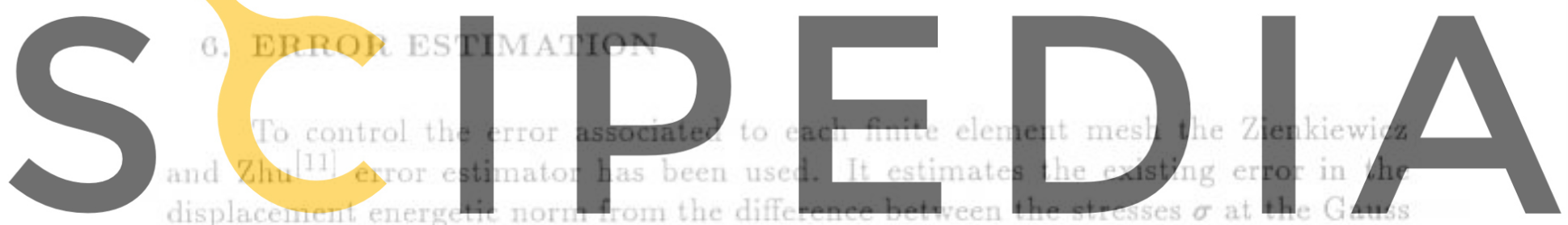

Register for free at https//www.scipedia.com to download the version without the watermark

$$
\|\boldsymbol{e}\|_{E}^{2} \approx \eta^{2}=\int_{\Omega}\left(\sigma^{*}-\sigma\right)^{T} D^{-1}\left(\sigma^{*}-\sigma\right) d \Omega=\sum_{\epsilon} \eta_{e}^{2}
$$

The last calculations can be performed element by element giving the error on each element as:

$$
\|e\|_{E_{\epsilon}}^{2} \approx \eta_{e}^{2}=\int_{\Omega_{e}}\left(\sigma^{*}-\sigma\right)^{T} D^{-1}\left(\sigma^{*}-\sigma\right) d \Omega
$$

With the values obtained from a given mesh it is possible to compute the necessary element sizes of a new mesh, which produces the required error level, using and AMR strategy. This error estimator has revealed to be quite robust, reliable and inexpensive, specially for linear elements. 


\section{SENSITIVITY ANALYSIS}

For each design it is necessary to compute the sensitivities of the objective function and restrictions. The sensitivity analysis for the whole problem is done step by step following the same path that the structural analysis. This path indicates the dependence of each quantity used in the structural analysis with respect to the rest of the quantities previously employed. For example, the expression of the stiffiness matrix depends on the nodal coordinates, so that, following the chain rule for derivatives, the stiffness matrix sensitivities can be expressed in terms of the nodal coordinates sensitivities. Then, it is necessary to compute the nodal coordinates sensitivities (mesh sensitivities) previously to the stiffness matrix sensitivities.

In the present implementation of the proposed methodology first and second order sensitivity analysis have been used. This sensitivity analysis has been developed to get directional derivatives of any quantity. So, in the next paragrafs the vector $s$ will mean a unity vector in the design variables space, and derivatives will be computed in the $s$ direction. To obtain the sensitivities with respect to a specific design variable $x_{i}$, the $s$ vector has to be the unity vector corresponding to the $x_{i}$ direction.

\subsection{B-spline sensitivity analysis}

The first order sensitivities of the B-splines can be obtained by derivation of eq. (2):

$$
\frac{\partial \mathbf{r}(t)}{\partial \boldsymbol{s}}=\sum_{l=0}^{q} \frac{\partial \mathbf{r}_{l}}{\partial \boldsymbol{s}} N_{4, l+1}(t)
$$

The terms $\partial \mathbf{r}_{l} / \partial \boldsymbol{s}$ can be extracted from the $\partial R / \partial s$ matrix obtained by derivation of equation (3):

$$
\frac{\partial R}{\partial s}=N^{-1}\left(\frac{\partial B}{\partial s}-\frac{\partial N}{\partial s} R\right)
$$

Second order sensitivities can be obtained using similar expressions:

$$
\frac{\partial^{2} \boldsymbol{r}(t)}{\partial \boldsymbol{s}^{2}}=\sum_{l=0}^{q} \frac{\partial^{2} \boldsymbol{r}_{l}}{\partial \boldsymbol{s}^{2}} N_{4, l+1}(t)
$$




$$
\frac{\partial^{2} R}{\partial s^{2}}=N^{-1}\left(\frac{\partial^{2} B}{\partial s^{2}}-\frac{\partial^{2} N}{\partial s^{2}} R-2 \frac{\partial N}{\partial s} \frac{\partial R}{\partial s}\right)
$$

Eqs. (15) and (17) give also the sensitivities of the nodes placed at the boundary of the domain fixing $t$ to the corresponding value.

\subsection{Mesh sensitivity analysis}

Once the sensitivites of the coordinates of each boundary node are known, it is also possible to compute the sensitivities of the coordinates of each internal nodal point (mesh sensitivities).

For non structured meshes its sensitivity analysis can be obtained by studying the variations of the mesh in front of changes in the design variables.

There are many different ways to define the movement of the mesh in terms of the design variables. One possibility is to consider that after a change in the boundary shape, the mesh nodes behave as part of a continuous elastic media moving towards a new equilibrium state. With this approach used by Wei Hong Zhang [9] the obtainment of the sensitivity of a mesh with respect to a specific design variable implies the resolution of a new linear elastic problem were the displacements of the boundary nodes are fixed. In this case the computation of the mesh sensitivity with respect to each different design variable needs the resolution of a new linear elastic problem with the same mesh but with different boundary conditions this implying a high computational cost.

It is also possible to consider a simpler elastic media defining the mesh movement. This is the case of the "springs analogy" where each element side is considered as a spring connecting two nodes. The force produced by each spring is proportional to its length. The resolution of the equilibrium problem in the springs analogy is simpler than the corresponding to a continuous elastic media but, again, it is also necessary to solve a system of equations with the same number of degrees of freedom than in the continuous case.

The spring analogy equilibrium problem can be solved iteratively, as done in the present work, using the Laplacian smoothing which is a very frequently used technique to improve the quality of non structured meshes. It consists in the application of some cycles where, in turn, each interior node of the mesh is placed in the baricenter of the nodes connected with it. For each cycle the expression of the new position vector of each node $\boldsymbol{r}_{i}$ is:

$$
r_{i}=\frac{\sum_{j=1}^{m_{i}} r_{j}}{m_{i}}
$$


where $r_{j}$ are the position vectors of the $m_{i}$ nodes connected with the $i$-th node.

To obtain the solution of the spring analogy equilibrium problem with a prescribed error tolerance it would be necessary to check the solution after each smoothing cycle. Taking into account that the described iterative process is only a way to obtain mesh sensitivities rather than the solution of the equilibrium problem itself, rigorous convergence conditions are not needed. For this reason we fix a priori the number of smoothing cycles to be applied. In the test cases we have checked, 50 loops are enough to assure a good quality of the results.

To obtain the first order mesh sensitivity analysis in any direction of the design variables space, $s$, it is necessary to derivate, for each cycle, eq. (19) with respect to $s$ :

$$
\frac{\partial \boldsymbol{r}_{i}}{\partial \boldsymbol{s}}=\frac{\sum_{j=1}^{m_{i}} \frac{\partial \boldsymbol{r}_{j}}{\partial \boldsymbol{s}}}{m_{i}}
$$

Second and higher order mesh sensitivities can be obtained by suecessive derivation of eq. (19):

$$
\frac{\partial^{2} \boldsymbol{r}_{i}}{\partial \boldsymbol{s}^{2}}=\frac{\sum_{j}^{m_{i}} \frac{\partial^{2} \boldsymbol{r}_{j}}{\partial \boldsymbol{s}^{2}}}{m_{i}}
$$

\subsection{Structural sensitivity analysis}

To get the structural analysis sensitivities it is necessary the derivation of the integral expressions (9), (10) and (12). To do that, we need to transform those integrals to the isoparametric space of the canonic coordinates $[5,6]$ where the shape of the integration domain does not depend on the design variables, and the derivation of an integral expression consists in the derivation of its kernel. The jacobian of this transformation $|J|$ can be expressed in terms of the node coordinates, so that, it can be derivated in order to know the integral sensitivities. Using the techniques developed in $[5,6]$ by Navarrina et al. the sensitivities of the elemental stiffness matrix can be obtained as:

$$
\begin{gathered}
\frac{\partial K_{e}}{\partial \boldsymbol{s}}=\int_{\Omega_{\xi}}\left[\frac{\partial B^{T}}{\partial \boldsymbol{s}} D B|J|+B^{T} \frac{\partial D}{\partial \boldsymbol{s}} B|J|+B^{T} D \frac{\partial B}{\partial \boldsymbol{s}}|J|+\right. \\
\left.B^{T} D B \frac{\partial|J|}{\partial \boldsymbol{s}}\right] d \xi_{1} d \xi_{2}
\end{gathered}
$$


Where the sensitivity of the jacobian is:

$$
\frac{\partial|J|}{\partial s}=|J| t \vec{r}\left(J^{-1} \frac{\partial J}{\partial s}\right)
$$

In eq. (21) the matrix $B$ depends on the nodal coordinates of the element, so that, its sensitivities $\partial \boldsymbol{B} / \partial$ s can be obtained through the mesh sensitivities.

Normally, the sensitivities of the $D$ matrix will be zero unless a design variable affects the mechanical properties of the material.

With this technique it is possible to obtain the first and higher order sensitivities of the stiffness matrix $K$, the nodal forces vector $f$ and any other integral expression involved in the computations. The detailed expressions for the computation of the first and higher order sensitivity analysis can be found in $[3,5,6]$.

After the computation of eq. (21) the sensitivities of the displacement vector $\boldsymbol{a}$ can be calculated as:

$$
\begin{gathered}
\frac{\partial \boldsymbol{a}}{\partial \boldsymbol{s}}=K^{-1}\left[\frac{\partial f}{\partial \boldsymbol{s}}-\frac{\partial K}{\partial \boldsymbol{s}} a\right] \\
\frac{\partial^{2} \boldsymbol{a}}{\partial \boldsymbol{s}^{2}}=K^{-1}\left[\frac{\partial^{2} f}{\partial \boldsymbol{s}^{2}}-\frac{\partial^{2} K}{\partial \boldsymbol{s}^{2}} a-2 \frac{\partial K}{\partial \boldsymbol{s}} \frac{\partial \boldsymbol{a}}{\partial \boldsymbol{s}}\right]
\end{gathered}
$$

Eqs. (23) and (24) show that the inverse of the stiffness matrix is used for the computation of each sensitivity. If a direct solver is used for the structural analysis this matrix has already been factorized and each new sensitivity analysis needs only a new backsubstitution process, but not a complete resolution of a new system of equations. On top of that, it is not necessary to assemble the sensitivities of the stiffness matrix because they always appear multiplying a vector, and this products can be computed element by element.

The strain and stress sensitivities can be computed from eq. (11) as:

$$
\begin{gathered}
\frac{\partial \varepsilon}{\partial s}=\frac{\partial B}{\partial s} a+B \frac{\partial a}{\partial s} \\
\frac{\partial^{2} \varepsilon}{\partial s^{2}}=\frac{\partial^{2} B}{\partial s^{2}} a+2 \frac{\partial B}{\partial s} \frac{\partial a}{\partial s}+B \frac{\partial^{2} a}{\partial s^{2}} \\
\frac{\partial \sigma}{\partial s}=\frac{\partial D}{\partial s} \varepsilon+D \frac{\partial \varepsilon}{\partial s}
\end{gathered}
$$




$$
\frac{\partial^{2} \sigma}{\partial s^{2}}=\frac{\partial^{2} D}{\partial s^{2}} \varepsilon+\frac{\partial D}{\partial s} \frac{\partial \varepsilon}{\partial s}+D \frac{\partial^{2} \varepsilon}{\partial s^{2}}
$$

To obtain the sensitivities of the smoothed stresses it is necessary to compute previously the sensitivities of the mass matrix $M$ and the $\Phi$ vector of eq. (12). The above commented techniques for the integral expressions are used to compute this sensitivities. Finally, the sensitivities of the smoothed stresses are obtained as:

$$
\begin{gathered}
\frac{\partial \bar{\sigma}^{*}}{\partial \boldsymbol{s}}=M^{-1}\left[\frac{\partial \Phi}{\partial s}-\frac{\partial M}{\partial s} \bar{\sigma}^{*}\right] \\
\frac{\partial^{2} \bar{\sigma}^{*}}{\partial s^{2}}=M^{-1}\left[\frac{\partial^{2} \Phi}{\partial s^{2}}-\frac{\partial^{2} M}{\partial s^{2}} \bar{\sigma}^{*}-2 \frac{\partial M}{\partial s} \frac{\partial \bar{\sigma}^{*}}{\partial s}\right] \\
\frac{\partial \sigma^{*}}{\partial s}=\bar{N}^{T} \frac{\partial \bar{\sigma}^{*}}{\partial s} \\
\frac{\partial^{2} \sigma^{*}}{\partial s^{2}}=\bar{N}^{T} \frac{\partial^{2} \bar{\sigma}^{*}}{\partial s^{2}}
\end{gathered}
$$

The same previous comments about the factorization of the stiffness matrix and its sensitivities can be applied to the mass matrix.

\subsection{Sensitivity analysis of the objective function and restrictions}

Both the objective function and restrictions will be expressed in terms of other quantities previously computed during the structural analysis.

Normally, the objective function is related with the volume of the whole or some parts of the structure to be optimized. This volume can be obtained as the sum of the volume of each finite element, and it can be computed through a simple integral expression:

$$
V=\sum_{e} V_{e}=\sum_{e} \int_{\Omega_{e}} d \Omega=\sum_{e} \int_{\Omega_{\xi}}|J| d \xi_{1} d \xi_{2}
$$

The same techniques above commented for the computation of the integral expressions sensitivity analysis can be applied for the derivation of eq. (33). 
Normally, restrictions will be expressed in terms of displacements or stresses. In this case the expression of each restriction can be derivated using the chain rule and this derivatives can be expressed in terms of the previously computed sensitivities of displacements and stresses.

\subsection{Sensitivity analysis of the error estimator}

The error estimator used in the present methodology is the integral expression (14). Again, the previously commented techniques for the computation of the sensitivity analysis of integral expressions can be used for the sensitivity analysis of the crror estimator. The resulting expression for the first order sensitivities is:

$$
\begin{aligned}
\frac{\partial \eta_{e}^{2}}{\partial s}=\int_{\Omega_{\xi}}[ & \left(\frac{\partial \sigma^{*}}{\partial \boldsymbol{s}}-\frac{\partial \sigma}{\partial \boldsymbol{s}}\right)^{T} D^{-1}\left(\sigma^{*}-\sigma\right)|J|+ \\
& \left(\sigma^{*}-\sigma\right)^{T} \frac{\partial D^{-1}}{\partial \boldsymbol{s}}\left(\sigma^{*}-\sigma\right)|J|+ \\
& \left(\sigma^{*}-\sigma\right)^{T} D^{-1}\left(\frac{\partial \sigma^{*}}{\partial \boldsymbol{s}}-\frac{\partial \sigma}{\partial \boldsymbol{s}}\right)|J|+ \\
& \left.\left(\sigma^{*}-\sigma\right)^{T} D^{-1}\left(\sigma^{*}-\sigma\right)|J| t r\left(J^{-1} \frac{\partial J}{\partial \boldsymbol{s}}\right)\right] d \xi_{1} d \xi_{2}
\end{aligned}
$$

The second order sensitivity analysis of the error estimator is done by derivation of eq. (34) and its detailed expression can be found in [3].

In order to use an AMR strategy it is also necessary to compute the elemental and total strain energy. The values of this strain energy and its sensitivities can be approximated from the finite element solution:

$$
\begin{gathered}
\|\boldsymbol{u}\|_{E_{e}}^{2} \approx \boldsymbol{a}^{T} K_{e} \boldsymbol{a} \\
\frac{\partial\|\boldsymbol{u}\|_{E_{e}}^{2}}{\partial \boldsymbol{s}} \approx \frac{\partial \boldsymbol{a}^{T}}{\partial \boldsymbol{s}} K_{e} \boldsymbol{a}+\boldsymbol{a}^{T} \frac{\partial K_{e}}{\partial \boldsymbol{s}} \boldsymbol{a}+\boldsymbol{a}^{T} K_{e} \frac{\partial \boldsymbol{a}}{\partial \boldsymbol{s}} \\
\frac{\partial^{2}\|\boldsymbol{u}\|_{E_{e}}^{2}}{\partial \boldsymbol{s}^{2}} \approx \frac{\partial^{2} \boldsymbol{a}^{T}}{\partial \boldsymbol{s}^{2}} K_{e} \boldsymbol{a}+\boldsymbol{a}^{T} \frac{\partial^{2} K_{e}}{\partial \boldsymbol{s}^{2}} \boldsymbol{a}+\boldsymbol{a}^{T} K_{e} \frac{\partial^{2} \boldsymbol{a}}{\partial \boldsymbol{s}^{2}}+ \\
2 \frac{\partial \boldsymbol{a}^{T}}{\partial \boldsymbol{s}} \frac{\partial K_{e}}{\partial \boldsymbol{s}} \boldsymbol{a}+2 \frac{\partial \boldsymbol{a}^{T}}{\partial \boldsymbol{s}} K_{e} \frac{\partial \boldsymbol{a}}{\partial \boldsymbol{s}}+2 \boldsymbol{a}^{T} \frac{\partial \boldsymbol{K}_{e}}{\partial \boldsymbol{s}} \frac{\partial \boldsymbol{a}}{\partial \boldsymbol{s}}
\end{gathered}
$$




\section{DESIGN IMPROVEMENT}

Using the information supplied by the finite element computations and the sensitivity analysis it is possible to use any optimization algorithm for the resolution of the mathematical problem of minimization (1). In the present methodology we have used an algorithm developed by Navarrina ${ }^{[5]}$. It uses information about first and second order sensitivities of the objective function and restrictions. Using this data and the design variables values for the $k$-iteration $\left(x^{k}\right)$ the algorithm searches the modified values of those variables defining an improved design:

$$
x^{k+1}=x^{k}+\theta^{k} s^{k}
$$

where $s^{k}$ is a unity vector corresponding to a direction of change in the design variables space, and $\theta^{k}$ is the advance or amplification factor [5].

The algorithm computes the direction of change $s^{k}$ using a complete first order sensitivity analysis. Then, a second order sensitivity analysis is computed in the direction of $s^{k}$ and it is used to perform a line search in this direction to obtain the $\theta^{k}$ factor.

\section{DEFINITION OF THE MESH FOR THE NEW DESIGN}

After the definition of the new design $(k+1)$ it is necessary to construct on it a background grid which contains the information to build the new mesh. This background mesh is the one used for the old design $(k)$ whose coordinates are projected to the actual design $(k+1)$. This extrapolation is done using the previously computed coordinate sensitivities. The new position vector for each node $r^{k+1}$ is:

$$
\boldsymbol{r}^{k+1}=\boldsymbol{r}^{k}+\theta^{k} \frac{\partial \mathbf{r}^{k}}{\partial \boldsymbol{s}^{k}}+\frac{1}{2} \theta^{k^{2}} \frac{\partial^{2} \boldsymbol{r}^{k}}{\partial \boldsymbol{s}^{k^{2}}}
$$

Likewise, the error and the deformation energy estimated over each element can also be projected using the expressions:

$$
\begin{gathered}
\eta_{e}^{k+1^{2}} \approx \eta_{\epsilon}^{k^{2}}+\theta^{k} \frac{\partial \eta_{\epsilon}^{k^{2}}}{\partial \boldsymbol{s}^{k}}+\frac{1}{2} \theta^{k^{2}} \frac{\partial^{2} \eta_{e}^{k^{2}}}{\partial \boldsymbol{s}^{k^{2}}} \\
\|\boldsymbol{u}\|_{E_{e}}^{k+1^{2}} \approx\|\boldsymbol{u}\|_{E_{e}}^{k^{2}}+\theta^{k} \frac{\partial\|\boldsymbol{u}\|_{E_{e}}^{k^{2}}}{\partial \boldsymbol{s}^{k}}+\frac{1}{2} \theta^{k^{2}} \frac{\partial^{2}\|\boldsymbol{u}\|_{E_{e}}^{k^{2}}}{\partial \boldsymbol{s}^{k^{2}}}
\end{gathered}
$$

This projections give an approximation to the values of the error and the 
deformation energy that would be obtained if the next design was computed with the previous mesh suitably deformed. Then, before any computation on the new design we get information about the quality of the results that would be obtained on it, with a mesh equivalent to the one used in the last design. From this point of view, an "a posteriori" error estimator is converted, through the projection, in an "a priori" error estimator for the new design. This is one of the key points of the methodology described in this work because it allows to use remeshing techniques without repeating calculations on the successive designs.

The projections of the mesh, the error estimator and the strain energy close the optimization loop of figure 1. After this projections the situation is exactly the same that in a standard remeshing procedure after using a previous mesh.

Using the extrapolated values it is possible to compute the required element size in the new mesh over the zone corresponding to each element of the background mesh. This is made by means of a suitable "optimality criteria" for the error distribution over the new mesh and an AMR strategy. In this work the criteria defined in ${ }^{[3]}$ has been used, which establishes that the optimum mesh is the one that has a uniform distribution of the error level $\eta^{2}$ for unit area (uniform specific error). This criteria is different from the classical one which establishes that a mesh is optimum when all the elements contains the same amount of error $([1,2,11\})$. The justification of the uniform specific error distribution criteria can be found in $[3]$.

After the specification of the maximum allowable percentage $\gamma$ of the error norm with respect to the total strain energy $\gamma$, the element size $h$ to be used in the new mesh generation in the zone corresponding to each element of the background grid is defined using:

$$
\begin{aligned}
\|\boldsymbol{e}\|_{E} & \leq \frac{\gamma}{100}\|\boldsymbol{u}\|_{E} \\
h & =\frac{h_{e}}{\xi_{e}^{1 / p}} \\
\xi_{E} & =\frac{100 \eta_{e}}{\gamma\|u\|_{E}} \sqrt{\frac{\Omega}{\Omega_{e}}}
\end{aligned}
$$

where $h_{e}$ is the size of the corresponding element of the background grid, $\Omega_{e}$ is the volume for each background element, $p$ is the order of the polynomials of the shape functions and $\Omega$ is the volume of the whole domain. Using the eq. (41) and the extrapolated values from $(39)$ and $(40)$ the characteristics of the desired mesh for the new design are completely defined.

The value of $\xi_{\epsilon}$ has been limited to 1.5 in order to avoid a too fast diminution of the size of the elements between two consecutive iterations. Numerical experiments show that the designs requiring a large number of elements are, many times, far away from the optimal one, and it is not useful to dedicate a lot of computational effort to 
compute it. The reason for it is that the optimization process tends to avoid stress concentrations where a large number of elements are needed. So, many times the designs close to the optimum one do not require the most dense meshes.

\section{EXAMPLES}

\subsection{Optimization of a pipe cross section: First case.}

The structure to be optimized is the cross section of a pipe subjected to an internal pressure. Due to the symmetry of the problem only one quarter of the piece has been considered. The initial shape is shown in figure 4 . This shape has been defined using four definition points with discontinuous slope. The chosen design variables are the distance of the two outer definition points to the origin $\left(x_{1}, x_{4}\right)$, the slope of the curve at this points $\left(x_{2}, x_{5}\right)$ and the corresponding $f$ factors $\left(x_{3}, x_{6}\right)$. This shape optimization problem consists in finding the best shape for the external boundary keeping constant the inner radius.

The considered mechanical properties do not correspond to any specific material. We have used $E=100000 . \mathrm{Kg} / \mathrm{cm}^{2}$, and $\eta=0.3$ in a plane strain model. A uniform interior pressure $\left(1.0 \mathrm{Kg} / \mathrm{cm}^{2}\right)$ has been applied as shown in figure 4 .

The objective function is the total volume of the pipe. Along all the boundary the maximum value of the biggest principal stress has been restricted to $10.0 \mathrm{Kg} / \mathrm{cm}^{2}$.

The problem has been solved using triangular quadratic elements and limiting the maximum percentage of the error norm $\gamma$ to $1 \%$ of the total strain energy.

The algorithm converges after 7 iterations. The successive meshes and designs can be observed in figure 5 , and the evolution of the objective function, global percentage of error and number of elements for each mesh can be observed in figures 6,7 and 8 respectively.

It can be observed that the evolution of the process is completely satisfactory. The error norm is controlled and a logical final shape is obtained. This example shows the utility of the limitation of the $\xi_{e}$ factor (see eq. (41)) to 1.5 because otherwise the number of elements on the second design would be very large in order to control the corresponding error. With the limitation of $\xi_{e}$ the error norm for the first designs is larger, but the final result is the same, and obtained with a much smaller computational effort. 


\subsection{Optimization of a pipe cross section: Second case.}

In the previous case a very simple parametrization with a small number of design variables has been used. Furthermore, the shape of the initial design is similar to the optimal one. In order to check the reliability of the proposed methodology, the optimization problem of the same cross section has been solved, but now using a very different parametrization and initial design.

The initial shape and some of the design variables are shown in figure 9 . The defined design variables are 6 coordinates $\left(x_{1}, x_{4}, x_{7}, x_{8}, x_{13}, x_{14}\right), 6$ angles $\left(x_{2}, x_{5}\right.$, $\left.x_{9}, x_{11}, x_{15}, x_{17}\right)$ and the factors $f$ associated to this angles $\left(x_{3}, x_{6}, x_{10}, x_{12}, x_{16}\right.$, $x_{18}$ ). The objective function and boundary restrictions are the same that in the first case. New restrictions have been imposed over the pairs $x_{7}, x_{8}$ and $x_{13}, x_{14}$ to get the corresponding points moving along the symmetry axes of the figure. The rest of the characteristics of the problem are exactly the same that in the first case.

The algorithm converges after 16 iterations. The successive meshes and designs can be seen in figure 10 and the evolution of the objective function, global percentage of error and number of elements for each mesh can be observed in figures 11,12 and 13 , respectively.

Despite the parametrization complexity the final design is the same that in the first example. The convergence of the objective function is very fast and the percentage of error keeps below the specified $1 \%$ beyond the sixth iteration.

There is an increase of the number of elements around the point $A$ (see figure 9) in the last iterations of the process. This is due to the presence of a kink producing a singular point in A (see figure 10). This concentration of elements reveals the presence of this singularity and suggests to use a different parametrization with a higher degree of continuity at the boundary shape. This is a good example of how an AMR strategy can help the designer to discover singularities and to improve the parametrization of the problem.

\subsection{Optimization of a connecting rod}

This example was first solved in [3], but it has been also used by other authors $\left[4,10^{\circ}\right.$ to check different optimization methodologies.

Due to the symmetry of the problem only one quarter of the piece has been considered (see figure 14). The initial shape and the definition points are shown in figure 14. At the points $1,2,6,7$ and 13 , discontinuous slopes $\left(90^{\circ}\right)$ have been imposed.

Very simple hypothesis about the load acting on the structure have been assumed. It has been modelled a parabolic normal load in between points 4 and 6 , with a resulting load of $400 \mathrm{Kg}$. The considered material properties have been: Young modulus $\mathrm{E}=2100000 \mathrm{Kg} / \mathrm{cm}^{2}$ and Poisson ratio $\nu=0.3$. A plane stress model 
with 6 node triangular elements has been used. The error level has been limited to $5 \%$.

The following 10 design variables have been used:

- $x$ coordinates of points 7 and 8 .

- $y$ coordinates of points $8,9,10,11,12$, and 13 .

- $f$ factors corresponding to the points 7 and 13 .

The objective function is the weight of the connecting rod. The restriction on the maximum value of the Von Mises stresses is $2000 \mathrm{Kg} / \mathrm{cm}^{2}$. This restriction has been applied to all the nodes placed along the boundary. The thickness of the tail has been underlimited to $0.25 \mathrm{~cm}$, and point number 8 has been enforced to move along a $45^{\circ}$ straight line.

The algorithm converges after 11 iterations. The successive meshes and designs can be seen in figure 15, and the evolution of the objective function, global percentage of error and number of elements are presented in figures 16,17 and 18 respectively.

In the evolution of the objective function (figure 16) there is a very fast initial drop to a minimum value at the iteration nr. 2. This value corresponds to a non feasible design because there are stresses higher than the allowed maximum. After that point the objective function grows slightly, up to reach a feasible final design.

The highest error percentage is obtained in the iteration nr. 3 , but beyond the iteration nr. 8 it remains below the prescribed $5 \%$ value. The high error levels in the initial iterations are due to the limitation of the $\xi_{e}$ (eq. (41)) factor to 1.5 .

\section{CONCLUSIONS}

A general methodology to perform structural optimization analysis including automatic mesh generation, error estimation techniques and adaptivity has been presented. The following aspects of the methodology have been highlighted:

- A very general parametrization to solve very different problems without any modification of the optimization computer code.

- The use of cubic B-splines to represent the boundary shape.

- The use of non structured meshes and the computation of its sensitivity analysis.

- The use of an error estimator to check the quality of the results obtained for each design and the development of its sensitivity analysis.

- The projection of the error estimator from one design to the next in order to use an AMR strategy without any extra computations on the new design. 
The presented examples show the usefulness, robustness and reliability of the proposed approach.

Although all the presented examples are $2 \mathrm{D}$ problems, the generalization of the proposed methodology to $3 \mathrm{D}$ cases is straightforward.

\section{REFERENCES}

(1) Babuška, I, and Rheinboldt, W.C. - Error Estimates for Adaptive Finite Element Computations. - SIAM Journal Numerical Analysis, vol 15. No. 4 (1978)

[2] Babuška, I. and Rheinboldt, W.C. - Reliable Error Estimation and Mesh Adaptation for the Finite Element Method. - Computational Methods in Nonlinear Mechanics, pp. 67.108, Ed. J.T. Oden (1980)

[3] Bugeda, G. - Utilización de técnicas de estimación de error y generación automática de mallas en procesos de optimización estructural. Ph D. Thesis Universitat Politècnica de Catalunya (1990), (In Spanish)

[4] E. Hinton, M. Özakça and N. V. R. Rao - An integrated approach to structural shape optimisation of linearly elastic structures. Part I: General methodology. \& Part II:shape definition and adaptivity. Computational Structures Technology conference. Edinburgh, U.K. (1991).

(5) Navarrina, F. - Una metodologia general para optimización estructural en diseño asistido por ordenador. Ph. D. Thesis Universitat Politècnica de Catalunya (1987), (In Spanish)

[6] Navarrina, F., Bendito, E. and Casteleiro M. - High order sensitivity analysis in shape optimization problems. - Computer Methods in Applied Mechanics and Engineering, vol. 75, pp. 267-281 (1989)

17 Peraire, J. - A Finite Element Method for Convection Dominated Flows. - Ph. D. Thesis - University College of Swansea (1986)

[8] Peraire, J., Morgan, K. and Peiró, J. - Unstructured finite element mesh generation and adaptive procedures for CFD. - AGARD FDP: Specialist's Meeting, Loen, Norway (1989)

9] Wei Hong Zhang - Calcul des sensibilites et optimisation de forme par la methode des elements finis. Ph D. Université de Liège (1991)

10 Zienkiewicz, O.C. and Taylor R. L. - The Finite Element Method. Fourth Edition. Volume 1. Basic Formulation and Linear Problems. - Mc Graw Hill (1989)

[11] Zienkiewicz, O.C. and Zhu, J.Z. - A simple error estimator and adaptive procedure for practical engineering analysis. - International Journal for Numerical Methods in Engineering, vol. 24, pp. 337-957 (1987) 


\section{LIST OF FIGURES}

Figure 1. General scheme for the proposed methodology.

Figure 2. Spacing and update of the generation front.

Figure 3. Generation of a new element.

Figure 4. Initial shape and parametrization of a pipe cross section. First case.

Figure 5. Successive meshes and designs for the first pipe cross section problem.

Figure 6. Evolution of the objective function for the first pipe cross section problem.

Figure 7. Evolution of the global percentage of error for the first pipe cross section problem.

Figure 8. Evolution of the number of elements of each mesh for the first pipe cross section problem.

Figure 9. Initial shape and parametrization of a pipe cross section. Second case.

Figure 10. Successive meshes and designs for the second pipe cross section problem.

Figure 11. Evolution of the objective function for the second pipe cross section problem.

Figure 12. Evolution of the global percentage of error for the second pipe cross section problem.

Figure 13. Evolution of the number of elements of each mesh for the second pipe cross section problem.

Figure 14. Initial shape and parametrization of a connecting rod.

Figure 15. Successive meshes and designs for the connecting rod problem.

Figure 16. Evolution of the objective function for the connecting rod problem.

Figure 17. Exolution of the global percentage of error for the connecting rod problem.

Figure 18. Evolution of the number of elements of each mesh for the connecting rod problem. 


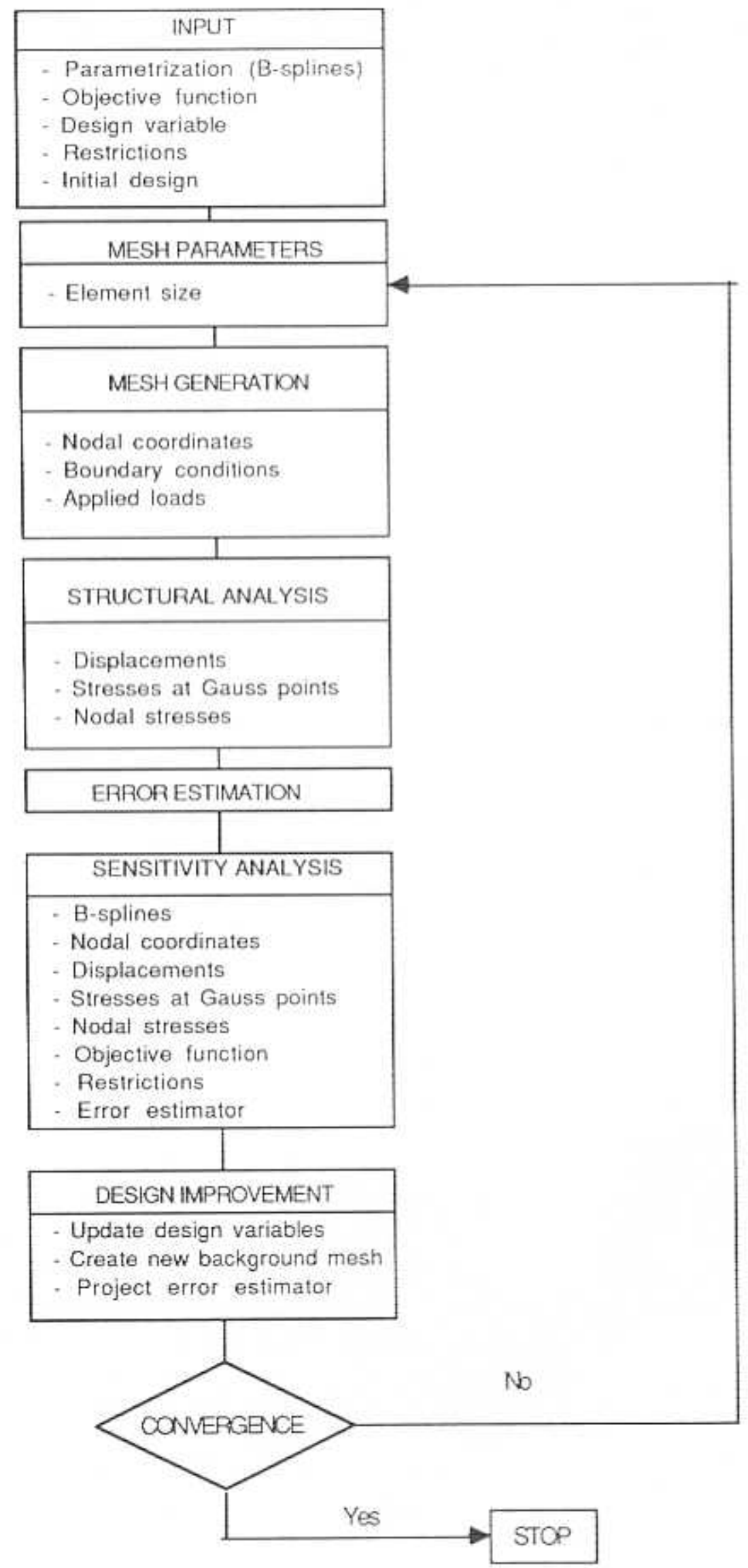

Figure 1. General scheme for the proposed methodology. 


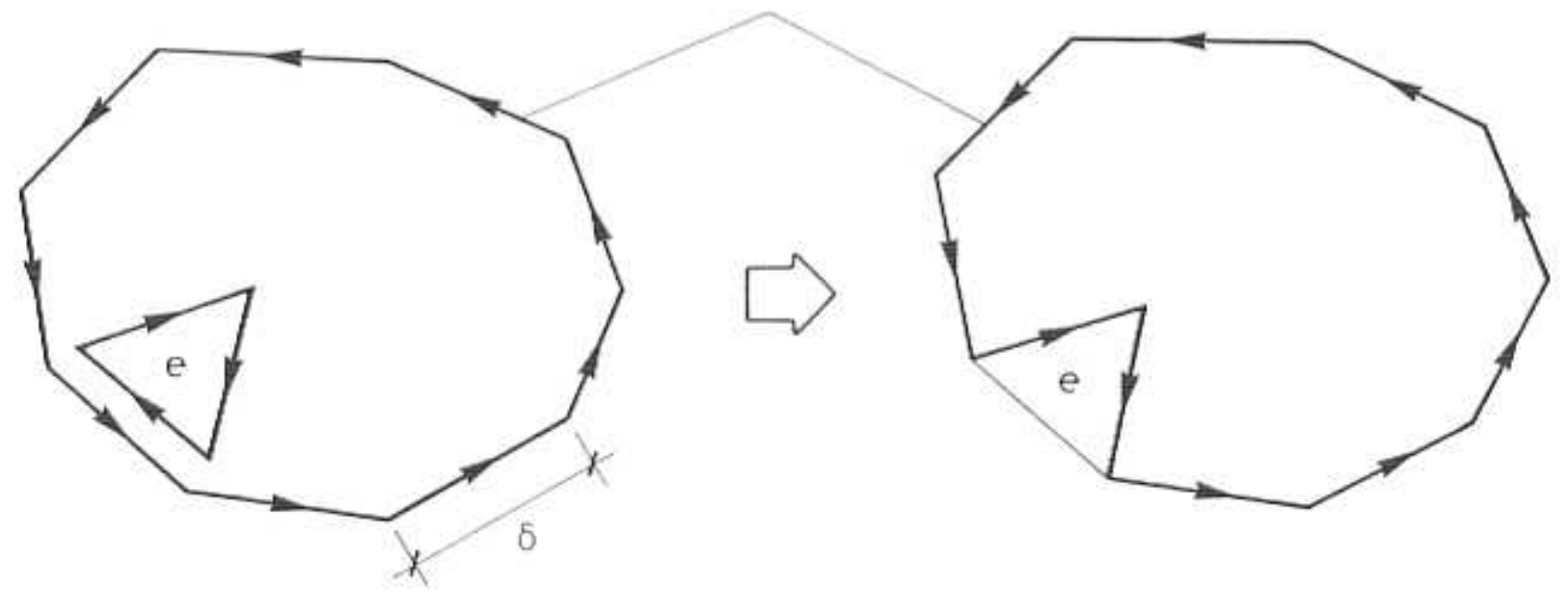

Figure 2. Spacing and update of the generation front. 


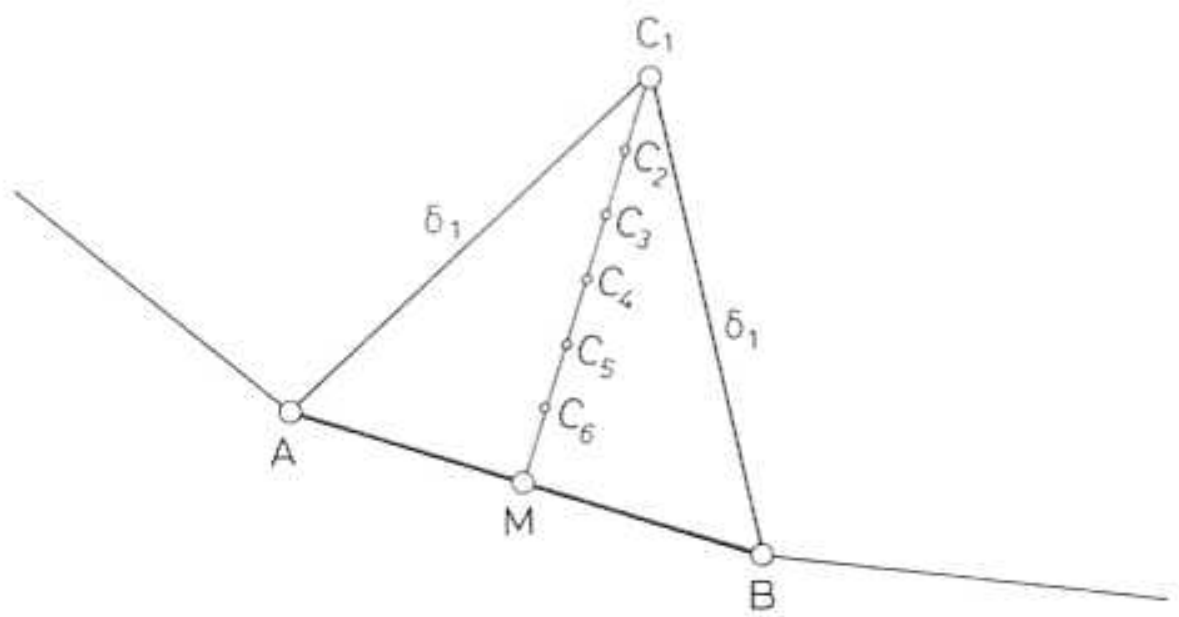

Figure 3. Generation of a new element. 


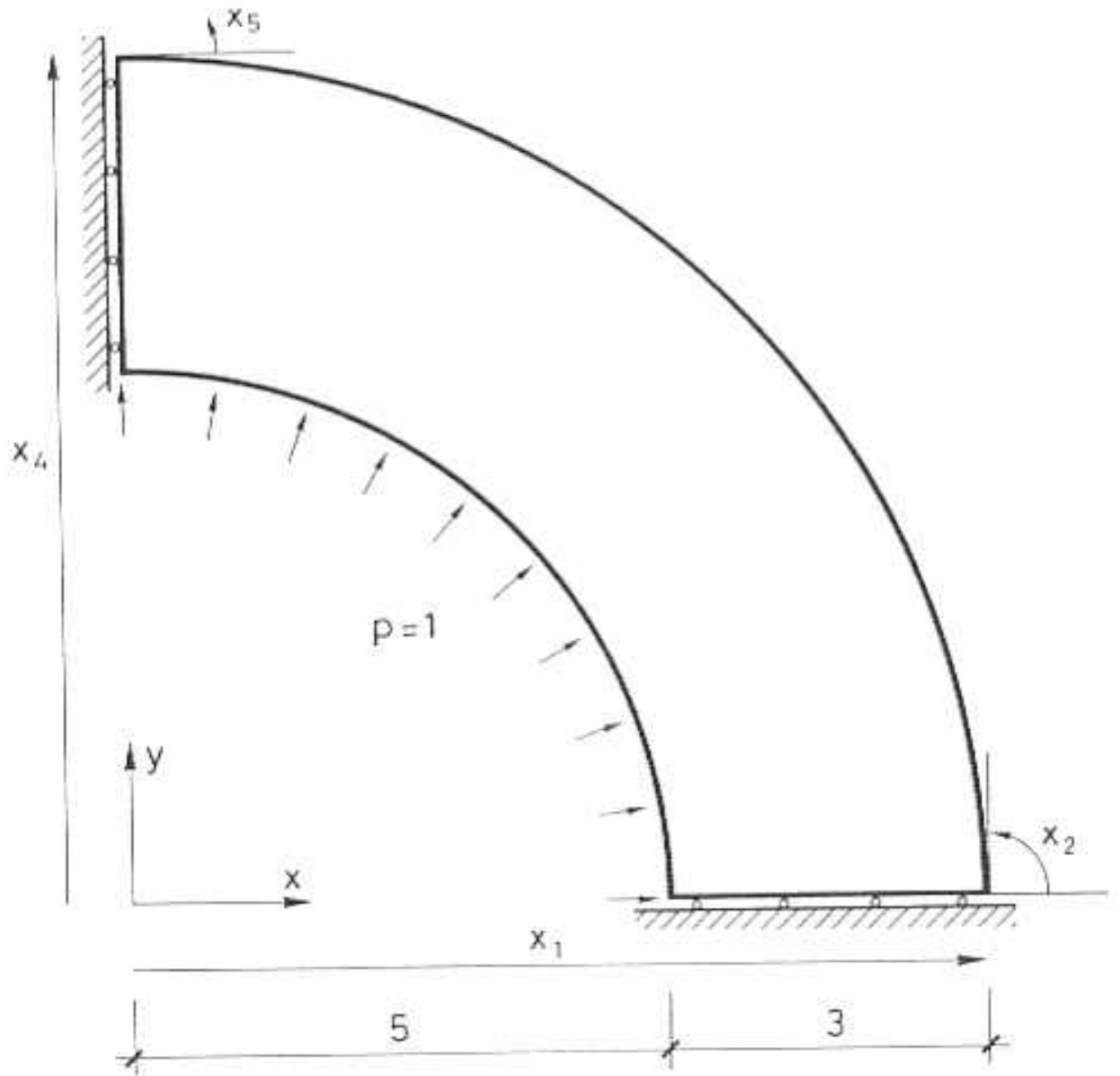

Figure 4. Initial shape and parametrization of a pipe cross section. First case. 


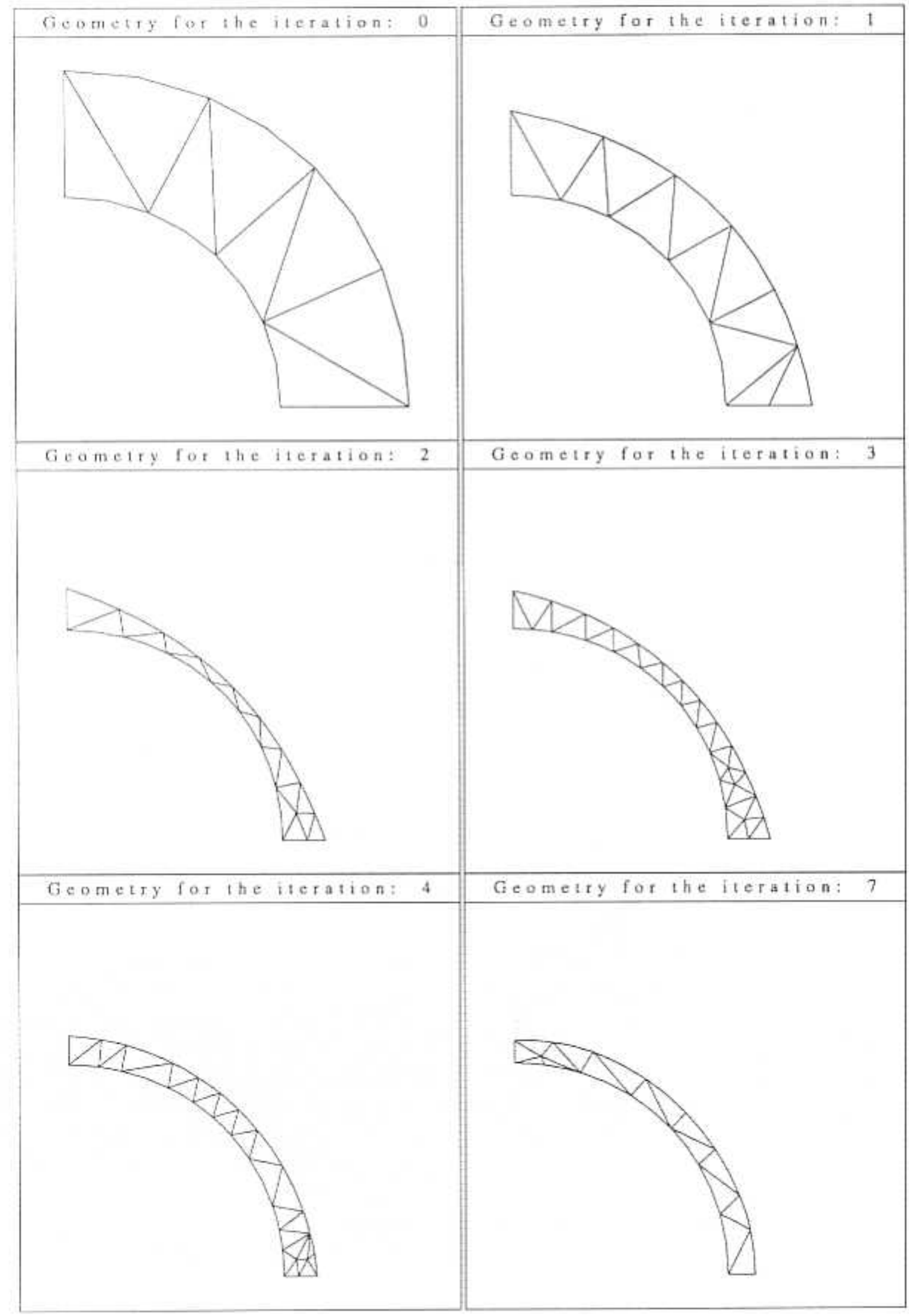

Figure 5. Successive meshes and designs for the first pipe cross section problem. 


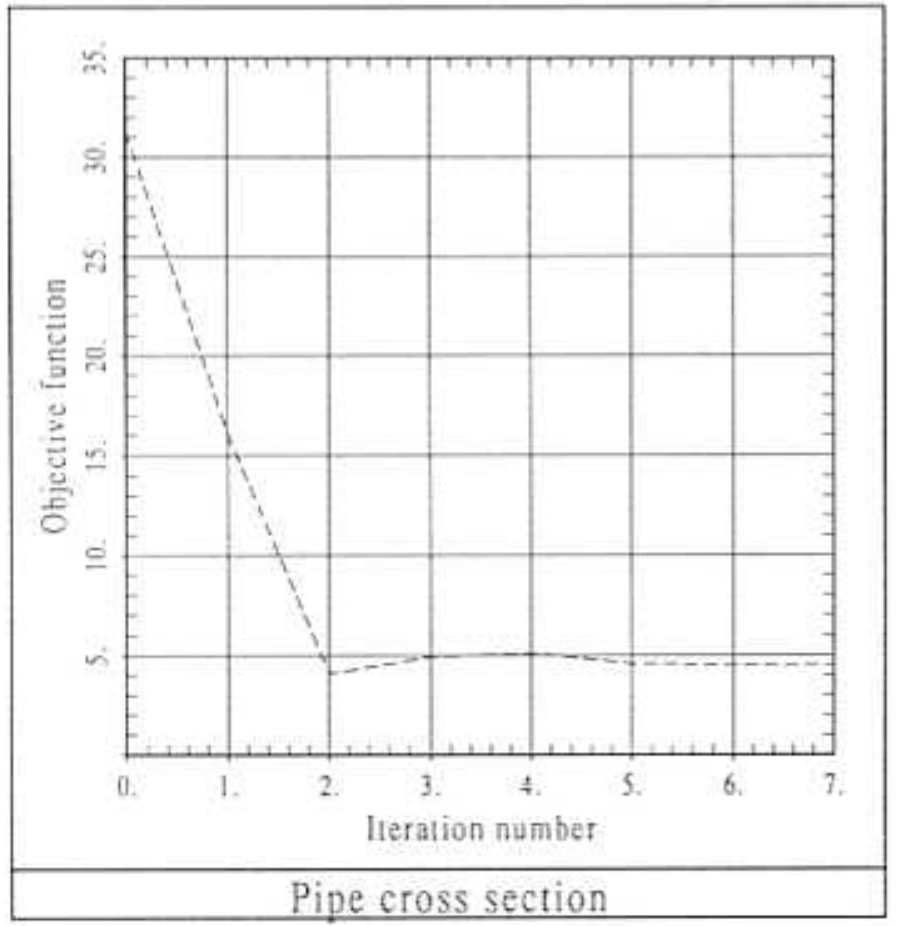

Figure 6.Evolution of the objective function for the first pipe cross section problem. 


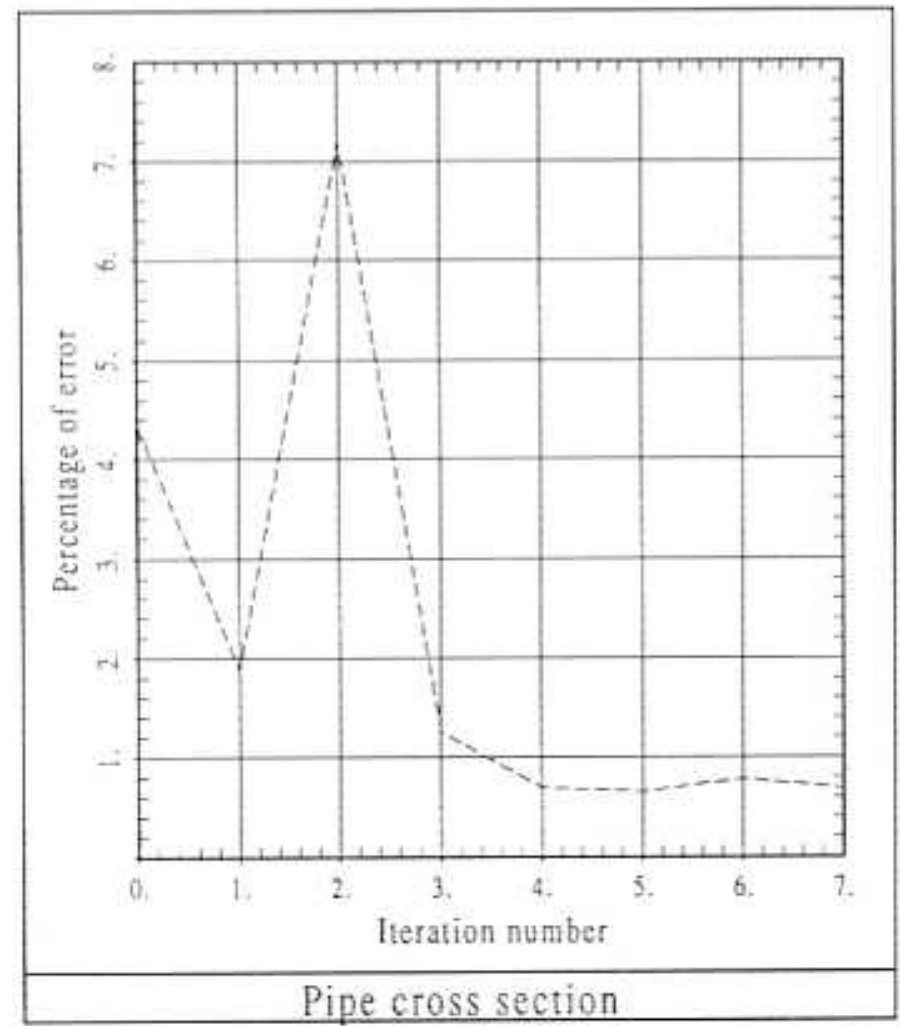

Figure 7.Evolution of the global percentage of errot for the first pipe cross section problem. 


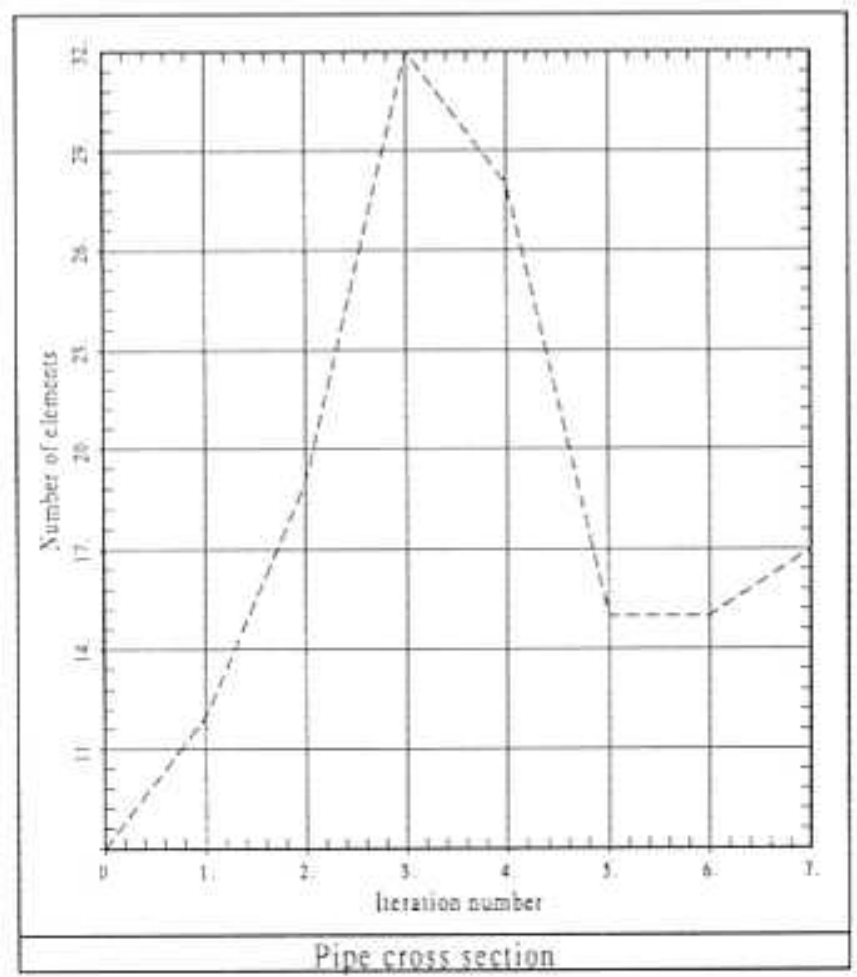

Figure 8. Evolution of the number of elements of each mesh for the first. pipe eross section problem. 


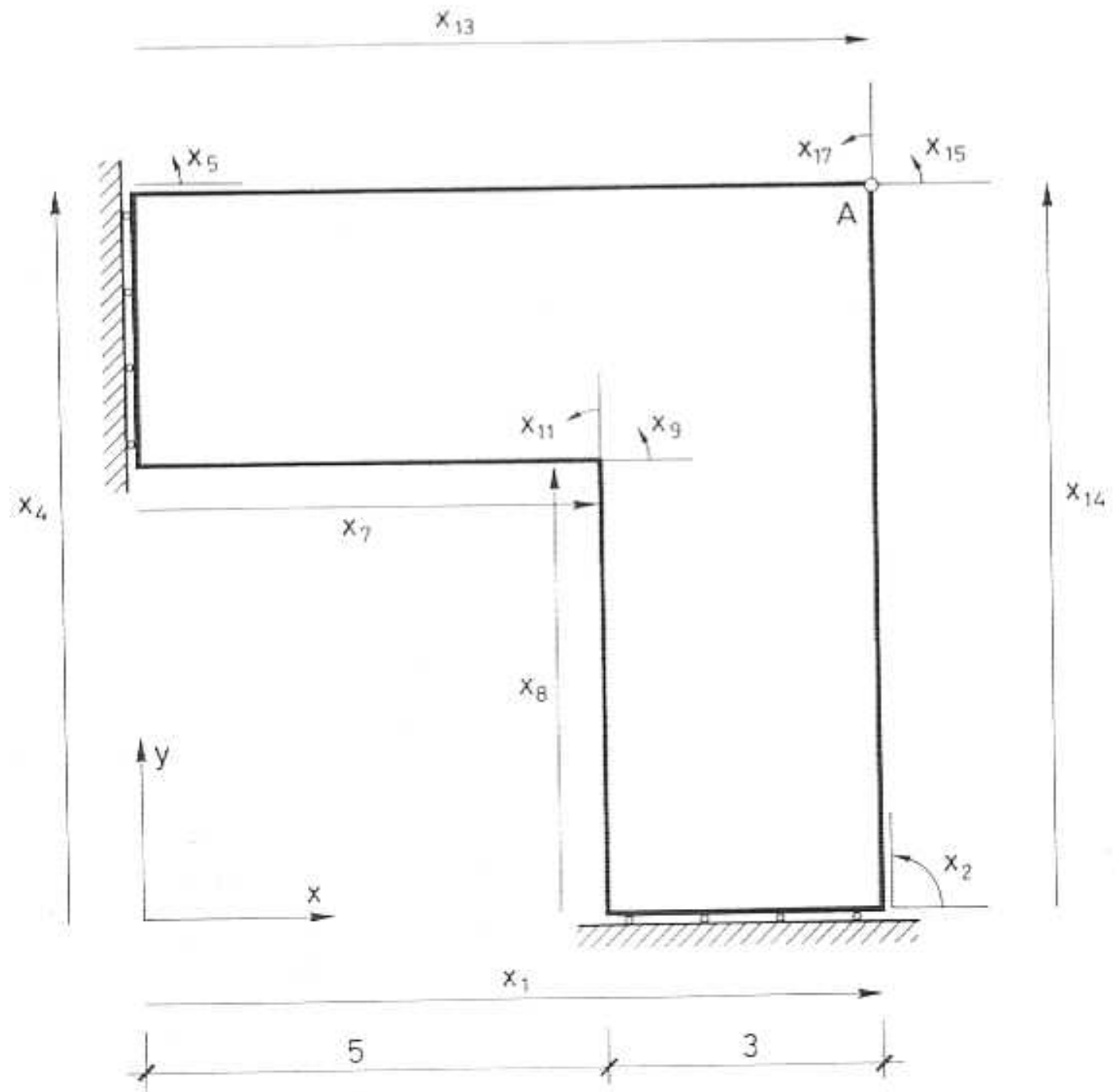

Figure 9.Initial shape and parametrization of a pipe cross section. Second case. 


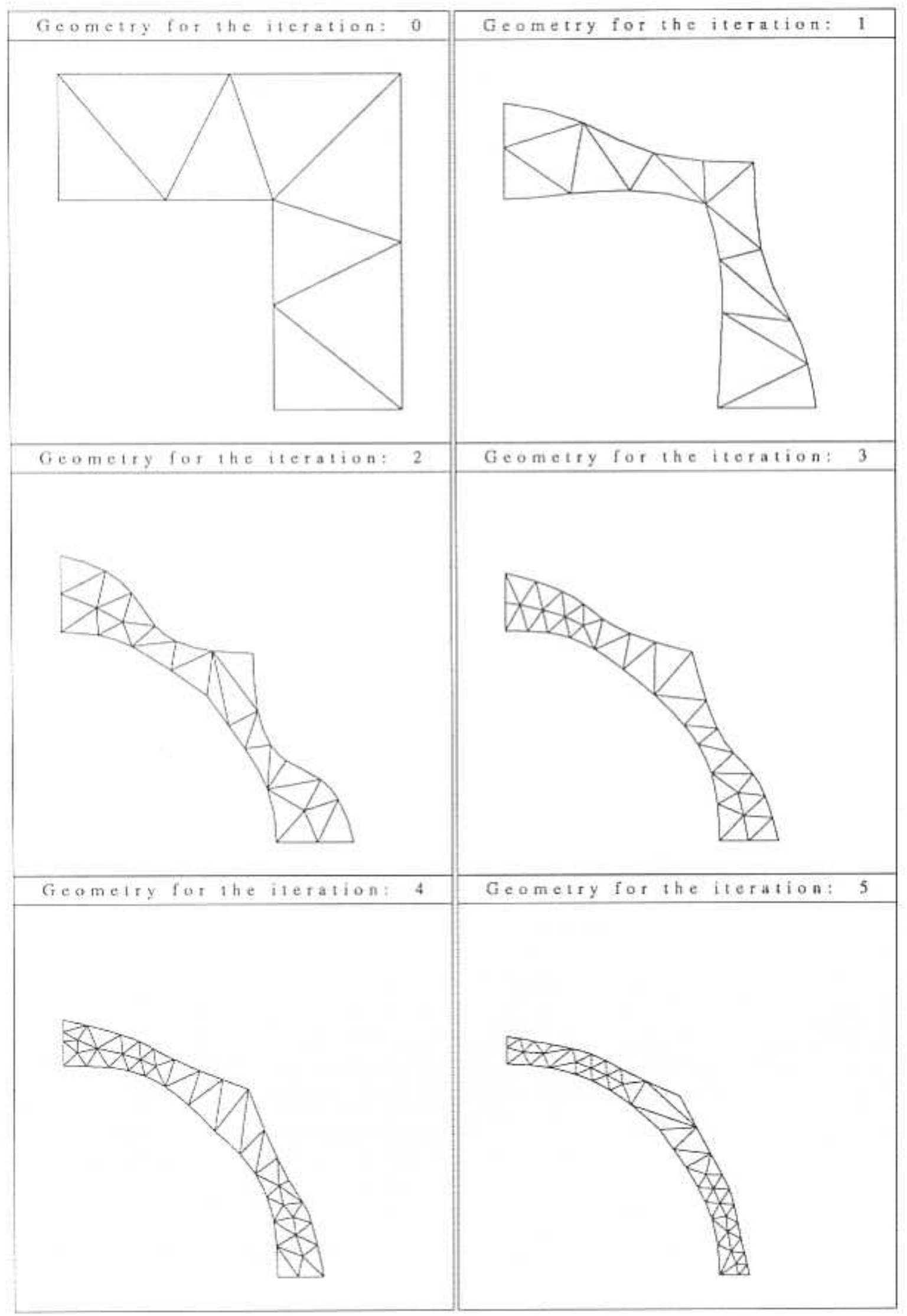

Figure 10A. Successive meshes and designs for the second pipe cross section problem. 


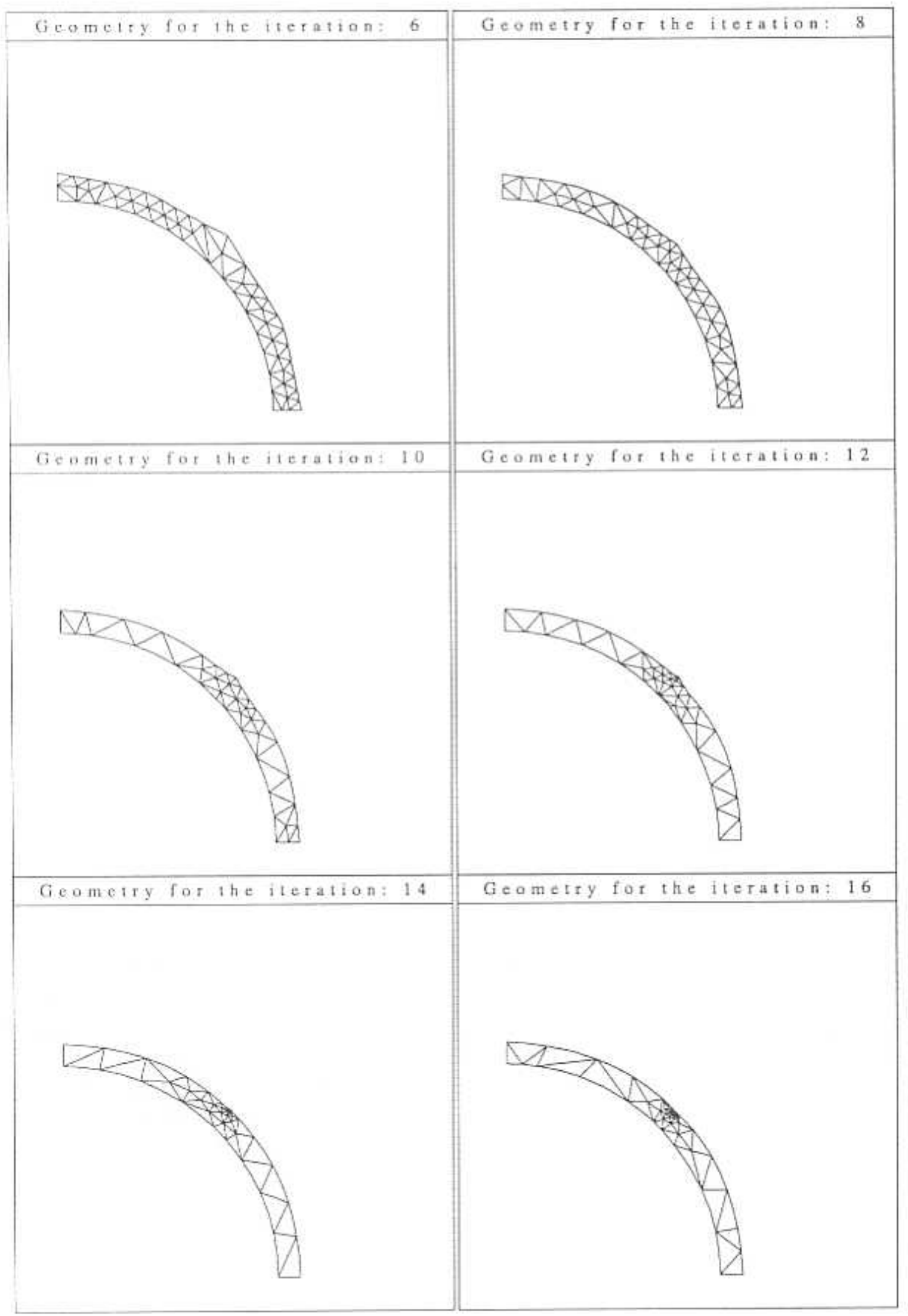

Figure 10B. Successive mestes and designs for the second pipe cross section problem. 


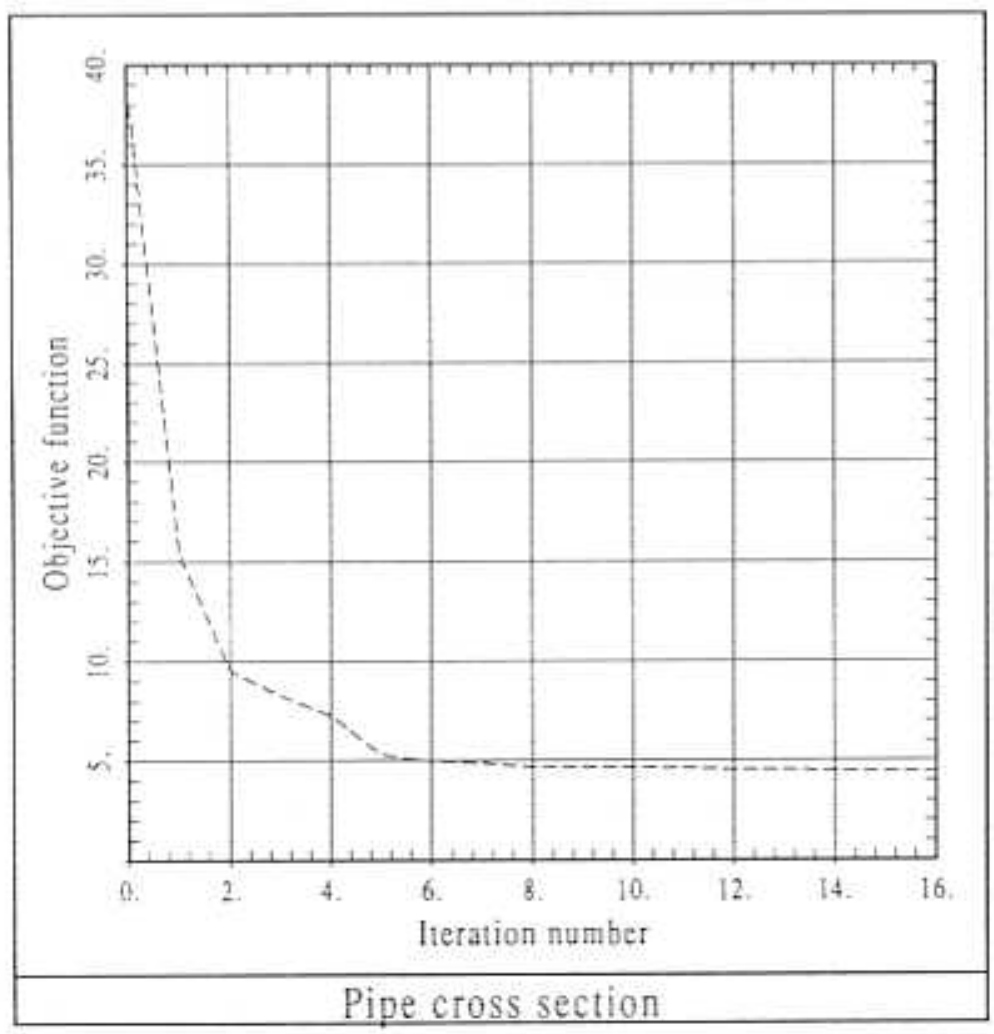

Figure 11.Evolution of the objective function for the second pipe cross section problem. 


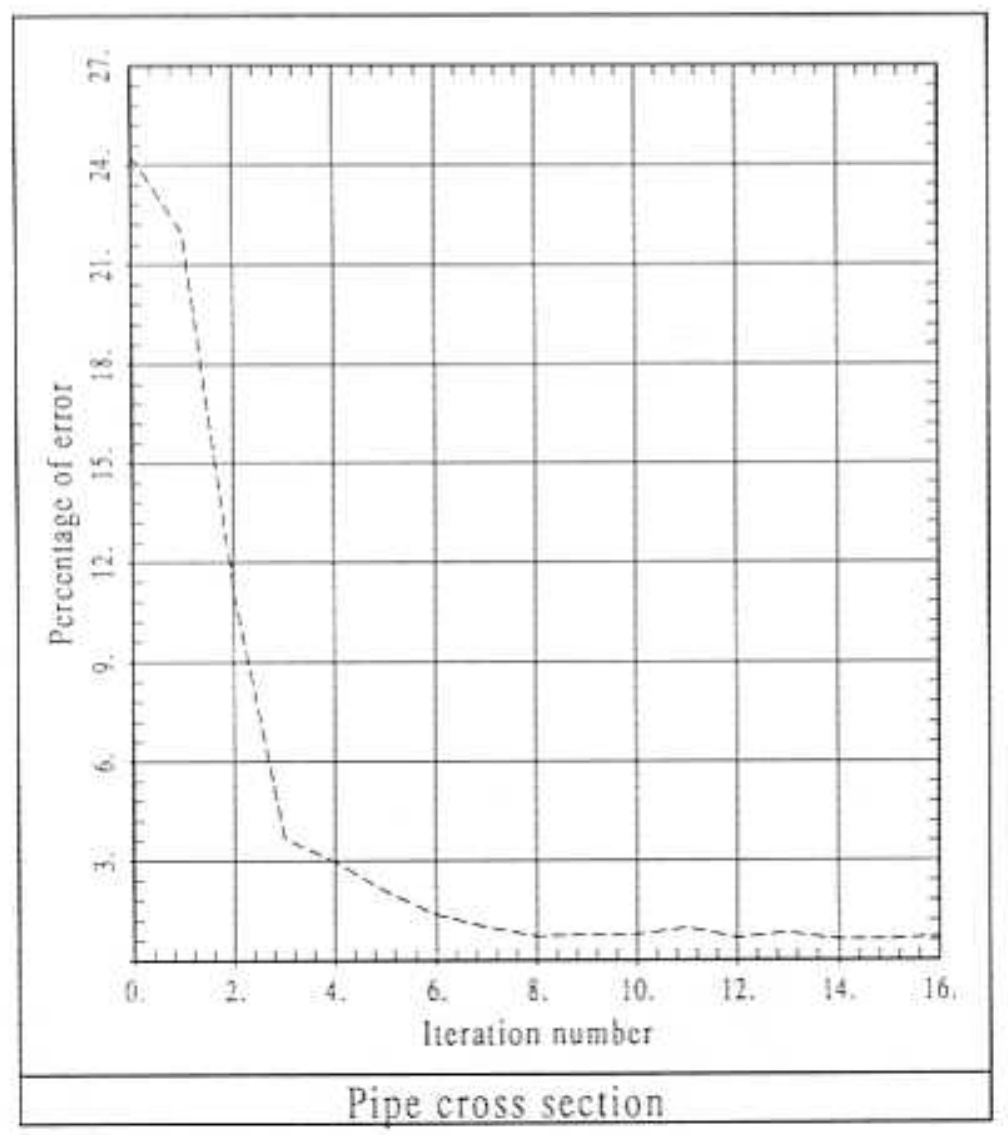

Figure 12.Evolution of the global percentage of ertor for the second pipe cross section problem. 


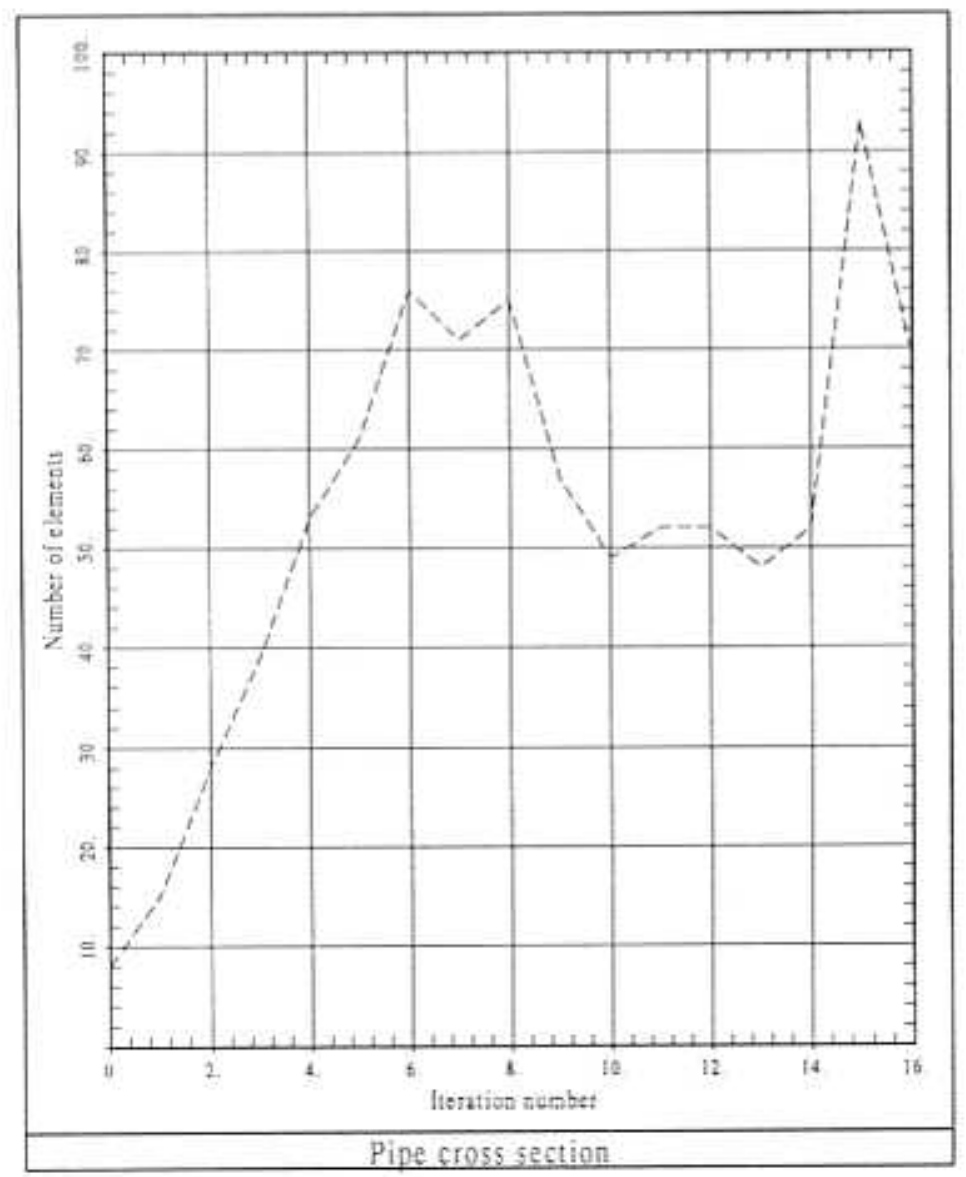

Figure 13.Evolution of the number of elements of each mesh for the second pipe cross section problem. 


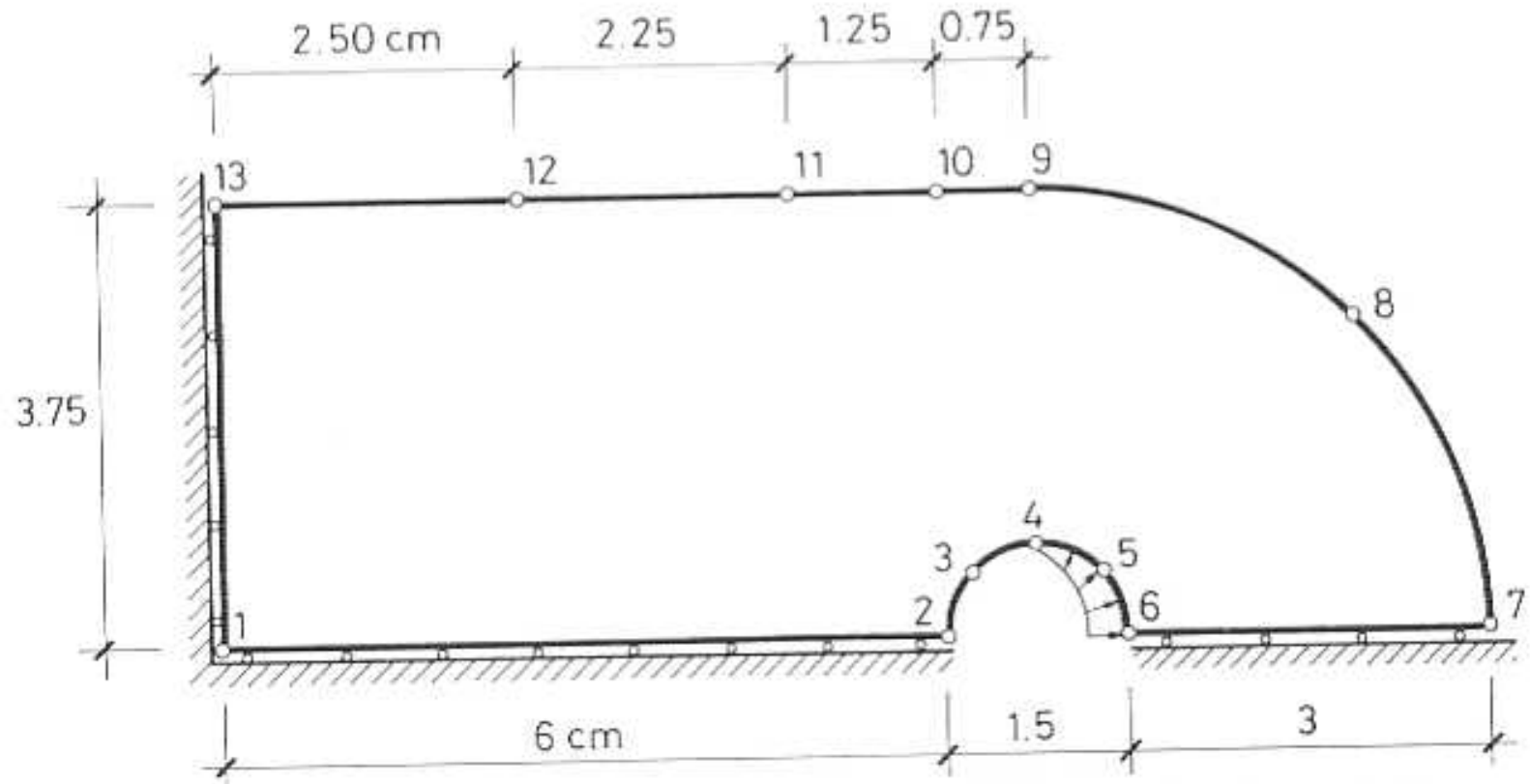

Figure 14. Initial shape and parametrization of a connecting rod. 


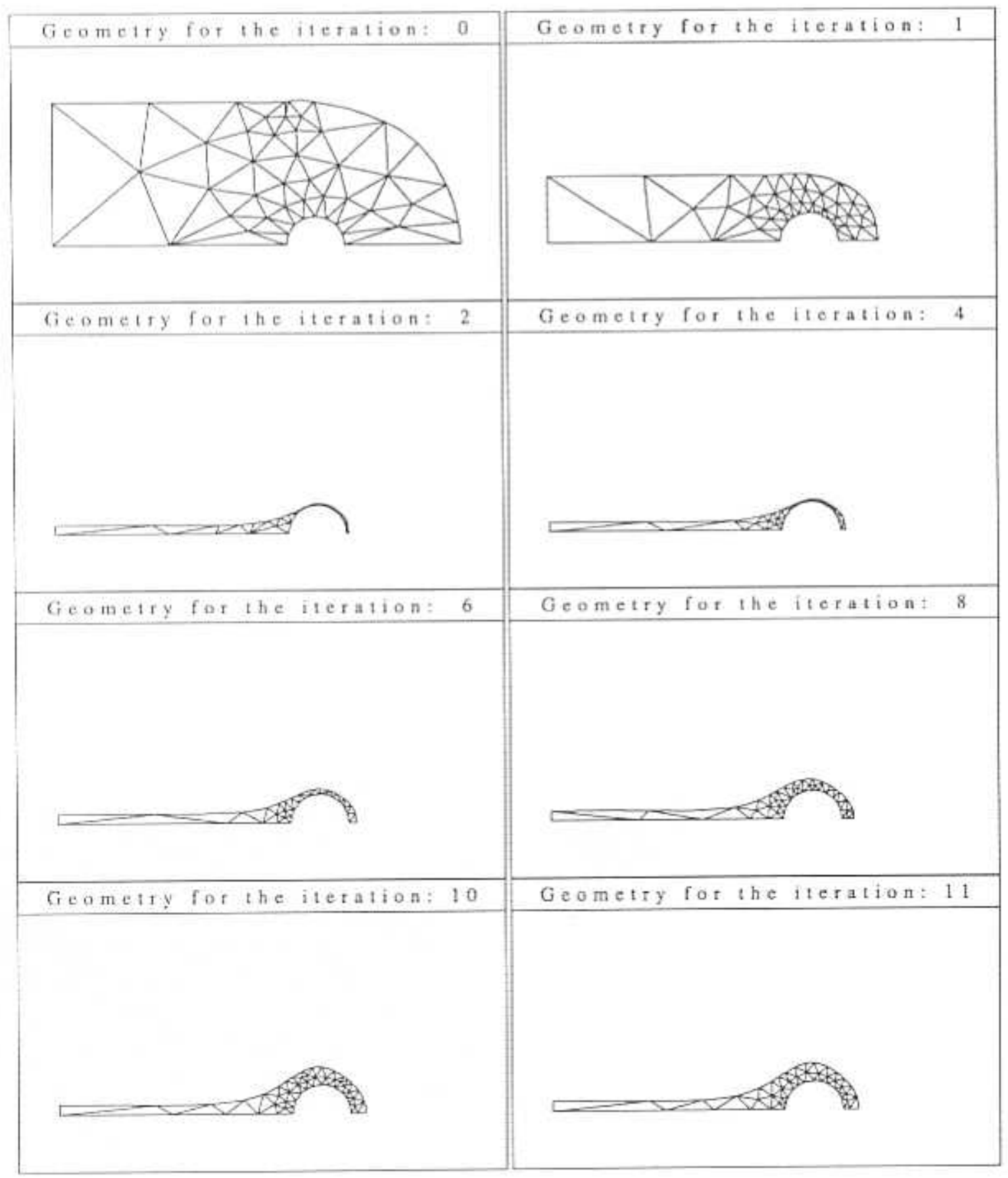

Figure 15. Successive meshes and designs for the connecting rod problem. 


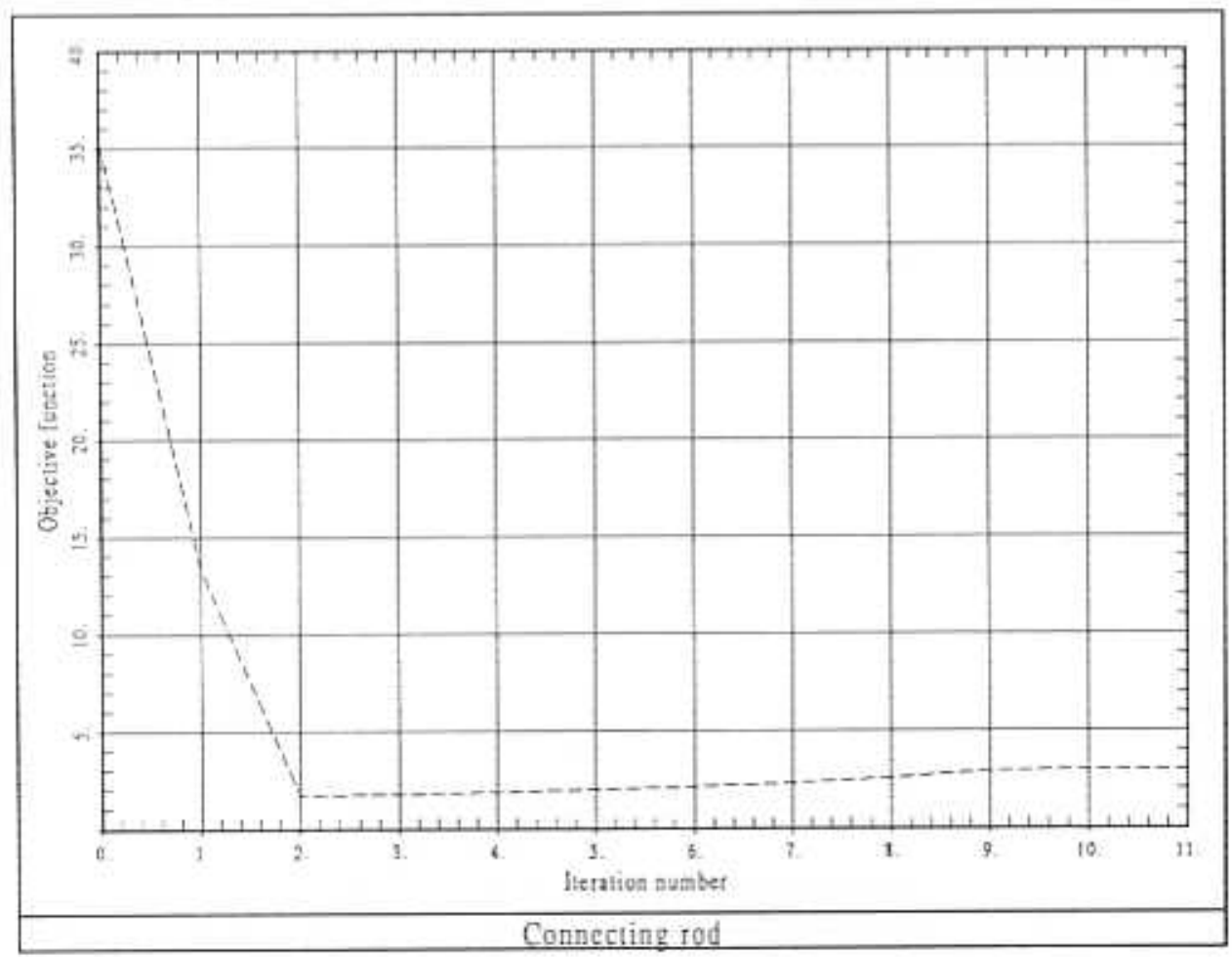

Figure 16. Evolution of the objective function for the connecting rod problem. 


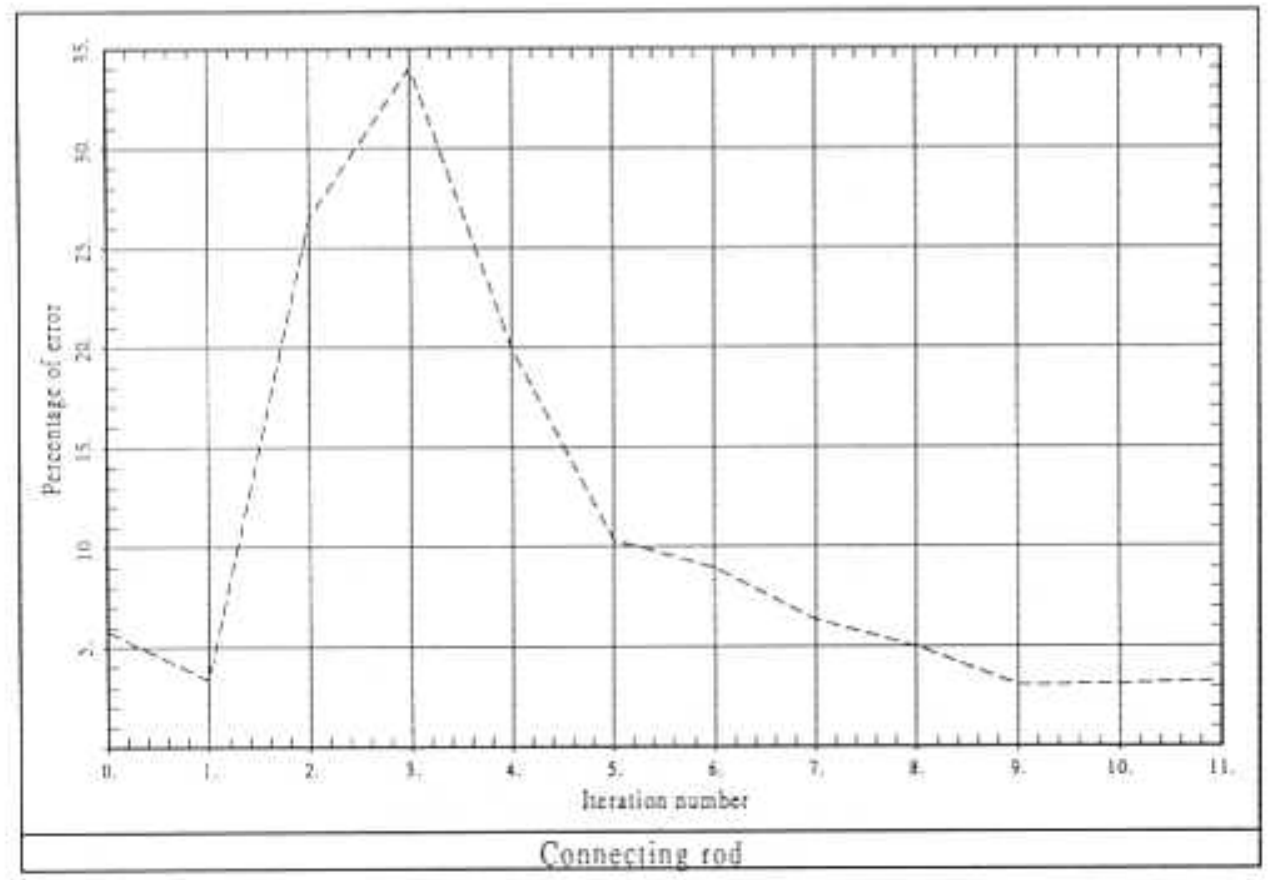

Figure 17.Evolution of the global percentage of error for the connecting rod problem. 


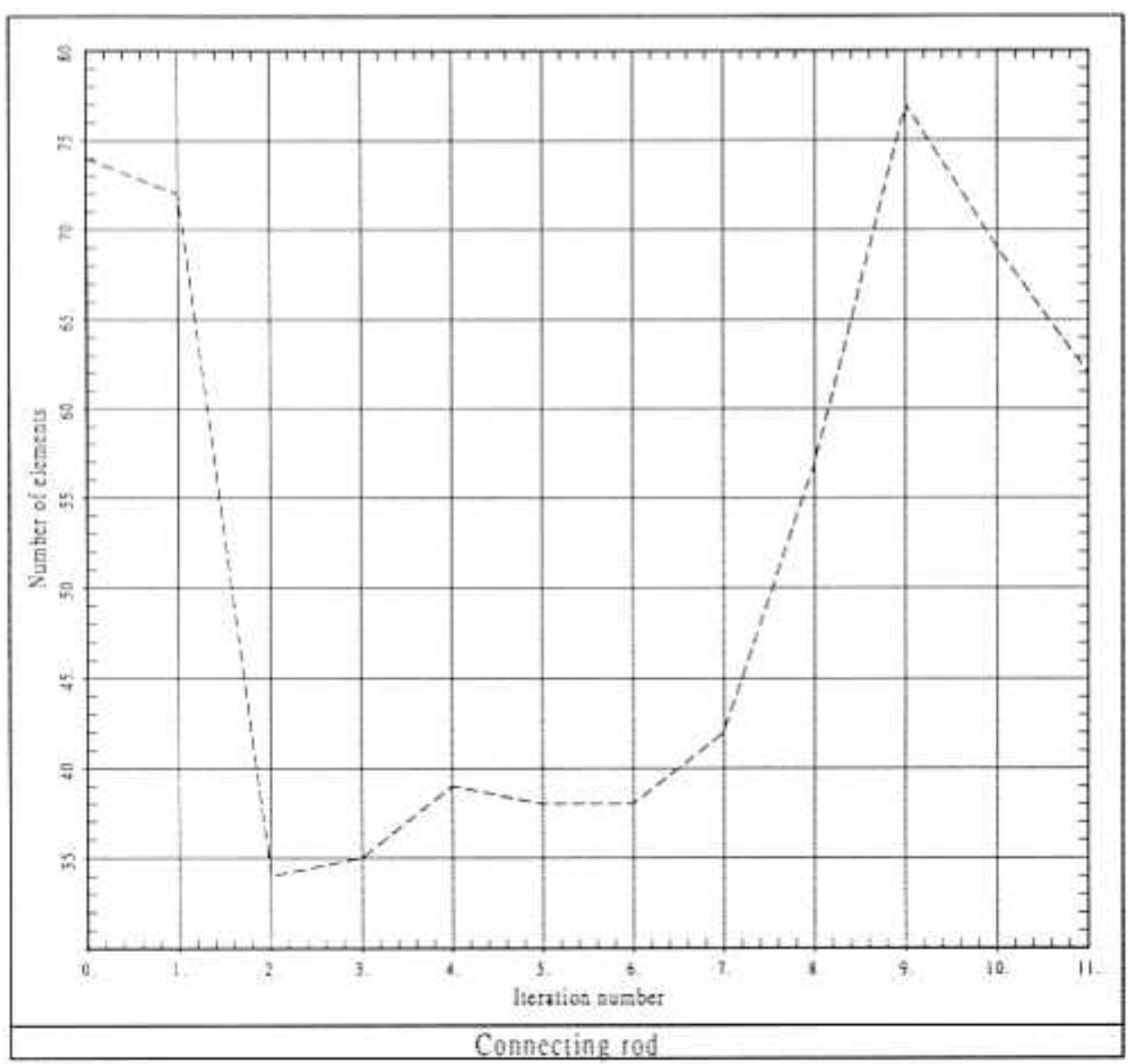

Figure 18. Evolution of the number of elements of each mesh for the connecting rod problem. 